\title{
Range-dependent adiabatic connections
}

A. M. Teale, , S. Coriani, and, and T. Helgaker

Citation: The Journal of Chemical Physics 133, 164112 (2010); doi: 10.1063/1.3488100

View online: http://dx.doi.org/10.1063/1.3488100

View Table of Contents: http://aip.scitation.org/toc/jcp/133/16

Published by the American Institute of Physics

\section{Articles you may be interested in}

Dispersion interactions in density-functional theory: An adiabatic-connection analysis

The Journal of Chemical Physics 135, 194109 (2011); 10.1063/1.3660357

Accurate calculation and modeling of the adiabatic connection in density functional theory

The Journal of Chemical Physics 132, 164115 (2010); 10.1063/1.3380834

A new mixing of Hartree-Fock and local density-functional theories

The Journal of Chemical Physics 98, 1372 (1998); 10.1063/1.464304

Long-range interactions from the many-pair expansion: A different avenue to dispersion in DFT

The Journal of Chemical Physics 146, 024111 (2017); 10.1063/1.4973728

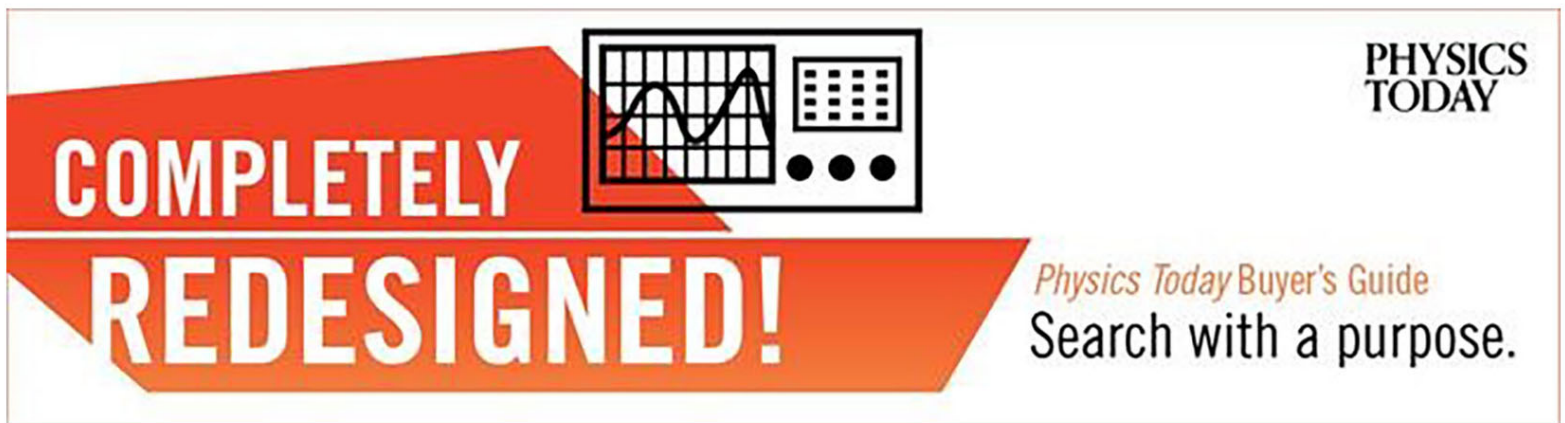




\title{
Range-dependent adiabatic connections
}

\author{
A. M. Teale, ${ }^{1, a)}$ S. Coriani, ${ }^{1,2}$ and T. Helgaker ${ }^{1}$ \\ ${ }^{1}$ Department of Chemistry, Centre for Theoretical and Computational Chemistry, University of Oslo, \\ P.O. Box 1033, Blindern, N-0315 Oslo, Norway \\ ${ }^{2}$ Dipartimento di Scienze Chimiche, Università degli Studi di Trieste, Via Licio Giorgieri 1, \\ I-34127 Trieste, Italy
}

(Received 11 May 2010; accepted 17 August 2010; published online 27 October 2010)

\begin{abstract}
Recently, we have implemented a scheme for the calculation of the adiabatic connection linking the Kohn-Sham system to the physical, interacting system. This scheme uses a generalized Lieb functional, in which the electronic interaction strength is varied in a simple linear fashion, keeping the potential or the density fixed in the process. In the present work, we generalize this scheme further to accommodate arbitrary two-electron operators, allowing the calculation of adiabatic connections following alternative paths as outlined by Yang [J. Chem. Phys. 109, 10107 (1998)]. Specifically, we examine the error-function and Gaussian-attenuated error-function adiabatic connections. It is shown that while the error-function connection displays some promising features, making it amenable to the possible development of new exchange-correlation functionals by modeling the adiabatic connection integrand, the Gaussian-attenuated error-function connection is less promising. We explore the high-density and strong static correlation regimes for two-electron systems. Implications of this work for the utility of range-separated schemes are discussed.
\end{abstract}

(C) 2010 American Institute of Physics. [doi:10.1063/1.3488100]

\section{INTRODUCTION}

The adiabatic-connection (AC) formula for the exchange-correlation energy ${ }^{1-5}$ in density-functional theory (DFT) has motivated the construction of orbital-dependent functionals ${ }^{6,7}$ which represent some of the most successful approximations in widespread use. The AC formula arises from a consideration of the link between the Kohn-Sham noninteracting system and the physical, interacting system as a function of the interaction strength. A number of studies have examined the AC using approximate methods, ${ }^{8-16}$ and some high-accuracy studies have been carried out for fewelectron atomic systems. ${ }^{17-22}$ Recently, we presented an implementation of a scheme allowing the calculation of accurate AC curves from $a b$ initio densities ${ }^{22,23}$ via optimization of Lieb functionals. ${ }^{24}$ Our implementation considers not only the usual density-fixed AC, of relevance in DFT, but also the potential-fixed $\mathrm{AC}^{22}$ of relevance in potentialfunctional theory (PFT) ${ }^{25}$ in which the fully interacting system is related to the noninteracting, bare-nucleus system (with the potential fixed at the external potential from the nuclei). The same connection was considered independently by Gross and Proetto, ${ }^{26}$ who also discussed the differences between potential-functional theory variants based on the bare-nucleus noninteracting system (as examined here) and potential-functional theories based on the Kohn-Sham noninteracting system as put forward by Yang and co-workers. ${ }^{25}$ The relationship between the density- and potential-fixed ACs is particularly clear from the point of view of the Lieb formulation of DFT and will be further elucidated in the present work.

a)Electronic mail: a.m.teale@kjemi.uio.no. FAX: +47 22855441.
Most previous studies of the AC consider only the case in which the electron-electron repulsion is modulated in a simple linear fashion, by introducing a straightforward scaling of the two-electron interaction. However, as was pointed out by Yang, ${ }^{27}$ this choice is not unique. In fact, the electronic interaction may be modified by any function that smoothly connects the noninteracting and physical systems. These generalized ACs are of particular relevance to theories constructed to combine the Kohn-Sham DFT and wavefunction approaches as proposed by Savin. ${ }^{28}$ With an appropriate modification of the electronic interaction, it is possible to attempt the construction of hybrid theories, in which short-range interactions are treated by DFT and long-range interactions by a suitable choice of wave-function methodology. Recently, a variety of short-range DFT functionals have been developed within the local-density approximation, ${ }^{28,29}$ the generalized gradient approximation (GGA), ${ }^{30-32}$ and the meta-GGA. ${ }^{33}$ Several implementations of these hybrid schemes exist combining short-range DFT functionals with long-range Hartree-Fock (HF), ${ }^{34}$ configuration-interaction (CI) ${ }^{35,36}$ second-order Møller-Plesset, ${ }^{34}$ coupled-cluster, $^{31}$ multiconfigurational self-consistent field, ${ }^{37,38}$ and $n$-electron valence second-order perturbation-theory ${ }^{39}$ methods. We also note that range separation of only the exchange interaction has been explored in the context of developing new DFT exchange-correlation functionals. Notable examples are the long-range corrected (LC) functionals developed by Hirao and co-workers, ${ }^{40,41}$ the $\omega$-PBEh and HSE functionals developed by Scuseria and co-workers, ${ }^{42,43}$ and the CAM-B3LYP functional developed by Handy and co-workers. ${ }^{44,45}$ These functionals emphasize either short-range $e^{42,43}$ or long-range $e^{40,41,44}$ interactions; a variant emphasizing the middle range has also been reported. ${ }^{46}$ Finally, in a different 
context, we note the use of a family of similar interactions by Gill and co-workers ${ }^{47-49}$ to remove the long-range tails of the Coulomb interaction.

While some studies of nonlinear ACs have been carried out from the point of view of calculating short-range DFT exchange-correlation energies and potentials, ${ }^{21,50}$ no direct studies of the generalized, range-dependent AC integrand have been presented, in contrast to the linear case. Given the central role that the AC formulation plays in the theory underlying range-separated approaches, we are motivated to consider the generalization of our previously introduced scheme to this task. These generalized ACs are also of a wider interest than range-separated methods. This point was clear in the work of Yang, ${ }^{27}$ who considered the complementary error function for modulation of the electronic interaction. By choosing an alternative form for the AC, the shape of the integrand is altered. For the complementary error function, this means that both the noninteracting and physical points are known to be simple constants. This behavior sharply contrasts the corresponding linear case, where the noninteracting point is the orbital-exchange energy functional of the Hartree-Fock theory and the interacting point an expectation value of the full CI (FCI) wave function.

In the present work, we generalize our optimization scheme for the Lieb functionals ${ }^{22}$ to electronic interactions weighted by the error function and the Gaussian-attenuated error function. We commence, in Sec. II, by introducing the theory of ACs with general two-electron operators and then briefly review our approach to optimization of the Lieb functionals and calculation of the AC integrands. Here we focus on details specific to this generalized scheme, referring the reader to our previous paper $^{22}$ and the work of $\mathrm{Wu}$ and Yang $^{23}$ for details of the optimization scheme. In Sec. III, we present results for the calculation of ACs corresponding to FCI densities for some simple two-electron systems, presenting potential-fixed as well as density-fixed connections. We also discuss the prospects for approximating these alternative connections by simple forms suitable for a self-consistent implementation. Finally in Sec. IV, we make some concluding remarks and discuss directions for future work.

\section{THEORY}

\section{A. Lieb's convex conjugate theory}

Consider an $\mathrm{N}$-electron system described by the Hamiltonian

$$
\hat{H}_{\lambda}[v]=\hat{T}+\hat{W}_{\lambda}+\sum_{i} v\left(\mathbf{r}_{i}\right), \quad 0 \leq \lambda \leq 1,
$$

where $v(\mathbf{r})$ is the external potential at $\mathbf{r}, \hat{T}$ is the kineticenergy operator

$$
\hat{T}=-\frac{1}{2} \sum_{i} \nabla_{i}^{2}
$$

and $\hat{W}_{\lambda}$ is a generalized electron interaction operator depending on a coupling-strength parameter $\lambda$ that varies between $\lambda=0$ (the noninteracting system) and $\lambda=1$ (the fully interacting system),

$$
\hat{W}_{\lambda}=\frac{1}{2} \sum_{i \neq j} w_{\lambda}\left(r_{i j}\right), \quad w_{0}\left(r_{i j}\right)=0, \quad w_{1}\left(r_{i j}\right)=1 / r_{i j} .
$$

We now introduce the ground-state energy $E_{\lambda}[v]$ as a functional of the external potential and the energy $F_{\lambda}[\rho]$ as a functional of the electron density by the following constrained minimizations ${ }^{24,51-53}$ over density matrices $\hat{\gamma}$ :

$$
\begin{aligned}
& E_{\lambda}[v]=\inf _{\hat{\gamma} \rightarrow N} \operatorname{Tr} \hat{H}_{\lambda}[v] \hat{\gamma}=\operatorname{Tr} \hat{H}_{\lambda}[v] \hat{\gamma}_{\lambda}^{v}, \\
& F_{\lambda}[\rho]=\inf _{\hat{\gamma} \rightarrow \rho} \operatorname{Tr} \hat{H}_{\lambda}[0] \hat{\gamma}=\operatorname{Tr} \hat{H}_{\lambda}[0] \hat{\gamma}_{\lambda}^{\rho},
\end{aligned}
$$

where we denote the minimizers by $\hat{\gamma}_{\lambda}^{v}$ and $\hat{\gamma}_{\lambda}^{\rho}$, respectively. Whereas a minimizer $\hat{\gamma}_{\lambda}^{\rho}$ always exists in Eq. (5), this is not so for the minimization in Eq. (4), where $\hat{\gamma}_{\lambda}^{v}$ only exists for those potentials $v$ that support an electronic ground state for a given interaction strength $\lambda$. In the following, we shall always assume that a minimizer exists.

As first discussed by Lieb, ${ }^{24}$ the ground-state energy as a functional of the external potential $E_{\lambda}[v]$ and the energy as a functional of the density $F_{\lambda}[\rho]$ are conjugate functionals (mutual Legendre-Fenchel transforms),

$$
\begin{aligned}
& E_{\lambda}[v]=\inf _{\rho \in X}\left(F_{\lambda}[\rho]+(v \mid \rho)\right), \\
& F_{\lambda}[\rho]=\sup _{v \in X^{*}}\left(E_{\lambda}[v]-(v \mid \rho)\right),
\end{aligned}
$$

where the domains $X$ and $X^{*}$ are reflexive Banach spaces such that $(v \mid \rho)=\int v(\mathbf{r}) \rho(\mathbf{r}) \mathrm{d} \mathbf{r}$ is finite for all $\rho \in X$ and $v$ $\in X^{*}$. In general, we obtain from Eqs. (6) and (7) the Fenchel inequality

$$
E_{\lambda}[v] \leq F_{\lambda}[\rho]+(v \mid \rho)
$$

which holds for all $v$ and $\rho$. In the absence of degeneracies, the conditions for a minimizing density $\rho$ in Eq. (6) and for a maximizing potential $v$ in Eq. (7) are equivalent and may be expressed in the following manner:

$E_{\lambda}[v]=F_{\lambda}[\rho]+(v \mid \rho) \Leftrightarrow \frac{\delta E_{\lambda}[v]}{\delta v(\mathbf{r})}=\rho(\mathbf{r}) \Leftrightarrow \frac{\delta F_{\lambda}[\rho]}{\delta \rho(\mathbf{r})}=-v(\mathbf{r})$,

where it is assumed that $\int \delta \rho(\mathbf{r}) \mathrm{d} \mathbf{r}=0$. An external potential $v$ and a density $\rho$ that together satisfy Eq. (9) are said to be conjugate. For a given potential $v$, one or more conjugate densities $\rho$ may be found provided the potential supports a (possibly degenerate) $N$-electron ground state. Conversely, an $N$-electron density $\rho$ has a conjugate potential $v$ (unique to within an additive constant) provided $\rho$ is $v$-representable. Substituting Eqs. (4) and (5) in Eq. (9), we note the relation

$$
\hat{\gamma}_{\lambda}^{v}=\hat{\gamma}_{\lambda}^{\rho} \quad(v \text { and } \rho \text { conjugate at } \lambda),
$$

which is valid for conjugate $v$ and $\rho$ in the absence of degeneracies. In the present work, we consider ACs in which we fix the (nondegenerate) density at its physical value (the $\mathrm{AC}$ of DFT) and an alternative connection, in which we fix the potential (the AC of PFT) at the nuclear-attraction potential since this potential corresponds to the $\lambda=1$ system. In 
other words, we consider connections that have conjugate $v$ and $\rho$ at $\lambda=1$.

\section{B. The adiabatic connection}

Let us now relate the functionals $E_{\lambda}[v]$ and $F_{\lambda}[\rho]$ for $\lambda>0$ to the corresponding noninteracting quantities $E_{0}[v]$ and $F_{0}[\rho]$, respectively,

$$
\begin{aligned}
& E_{\lambda}[v]=E_{0}[v]+\int_{0}^{\lambda} E_{\nu}^{\prime}[v] \mathrm{d} \nu, \\
& F_{\lambda}[\rho]=F_{0}[\rho]+\int_{0}^{\lambda} F_{\nu}^{\prime}[\rho] \mathrm{d} \nu,
\end{aligned}
$$

where the prime denotes differentiation with respect to $\nu$. On the right-hand side of these equations, we insert the expressions for the noninteracting energies $E_{0}[v]$ and $F_{0}[\rho]$ obtained by setting $\lambda=0$ in Eqs. (4) and (5). Next, we determine the derivatives $E_{\nu}^{\prime}[v]$ and $F_{\nu}^{\prime}[\rho]$ by differentiation of Eqs. (4) and (5) followed by application of the HellmannFeynman theorem, leading to the following AC expressions:

$$
\begin{aligned}
& E_{\lambda}[v]=H_{\mathrm{s}}[v]+\int_{0}^{\lambda} \mathcal{W}_{\nu}[v] \mathrm{d} \nu, \\
& F_{\lambda}[\rho]=T_{\mathrm{s}}[\rho]+\int_{0}^{\lambda} \mathcal{W}_{\nu}[\rho] \mathrm{d} \nu .
\end{aligned}
$$

We have here introduced the noninteracting bare-nucleus and kinetic-energy functionals

$$
\begin{aligned}
& H_{\mathrm{s}}[v]=\inf _{\hat{\gamma} \rightarrow N} \operatorname{Tr} \hat{H}_{0}[v] \hat{\gamma}=\operatorname{Tr} \hat{H}_{0}[v] \hat{\gamma}_{0}^{v}, \\
& T_{\mathrm{s}}[\rho]=\min _{\hat{\gamma} \rightarrow \rho} \operatorname{Tr} \hat{H}_{0}[0] \hat{\gamma}=\operatorname{Tr} \hat{H}_{0}[0] \hat{\gamma}_{0}^{\rho},
\end{aligned}
$$

and the potential- and density-fixed AC integrands as expectation values of the differentiated two-electron operator $\hat{W}_{\nu}^{\prime}$,

$$
\begin{aligned}
& \mathcal{W}_{\nu}[v]=\operatorname{Tr} \hat{W}_{\nu}^{\prime} \hat{\gamma}_{\nu}^{\nu}, \\
& \mathcal{W}_{\nu}[\rho]=\operatorname{Tr} \hat{W}_{\nu}^{\prime} \hat{\gamma}_{\nu}^{\rho},
\end{aligned}
$$

with respect to the density matrices $\hat{\gamma}_{\nu}^{v}$ and $\hat{\gamma}_{\nu}^{\rho}$ optimized at interaction strength $\nu$ from Eqs. (4) and (5), respectively. The perturbative expansion of Eqs. (11) and (12) in $\lambda$ leads to the bare-nucleus ${ }^{54}$ and Görling-Levy ${ }^{55,56}$ perturbation theories, respectively, as discussed in Ref. 22.

Let us now consider the relationship between the potential- and density-fixed connections. From Fenchel's inequality Eq. (8) applied at $\lambda=0$, we obtain

$$
H_{\mathrm{s}}[v] \leq T_{\mathrm{s}}[\rho]+(v \mid \rho) \quad(v \text { and } \rho \text { arbitrary }),
$$

where equality occurs when $\rho$ is conjugate to $v$ at $\lambda=0$. Substituting Eqs. (13) and (14) into the stationary condition Eq. (9) and invoking Eq. (19), we obtain the inequality

$$
\int_{0}^{\lambda} \mathcal{W}_{\nu}[v] \mathrm{d} \nu \geq \int_{0}^{\lambda} \mathcal{W}_{\nu}[\rho] \mathrm{d} \nu \quad(v \text { and } \rho \text { conjugate at } \lambda),
$$

which is valid provided $v$ and $\rho$ are conjugate at $\lambda$. Finally, introducing Eq. (10) in Eqs. (17) and (18), we note that $\mathcal{W}_{\nu}[v]=\mathcal{W}_{\nu}[\rho]$ when $v$ and $\rho$ are conjugate for interaction strength $\nu$, in the absence of degeneracies. In the present work, the potential-fixed connection has $v$ equal to the physical external potential due to the nuclei for all interaction strengths $\nu$, making it relevant to PFTs based on this external potential. An alternative PFT was recently discussed by Yang and co-workers, ${ }^{25}$ in which the energy is expressed as a functional of the Kohn-Sham potential. In the present context we note that construction of an AC for such a theory would mean using the maximizing potential of Eq. (7) at all $\nu$. This potential is different at each value of $\nu$ but always conjugate to the physical density. As a consequence this alternative potential-based AC and the density-fixed AC become identical.

From the concavity of $E_{\lambda}[v]$ and $F_{\lambda}[\rho]$ in $\lambda$, it follows that these functions can always be represented in the form of Eqs. (13) and (14), where the integrands $\mathcal{W}_{\nu}[v]$ and $\mathcal{W}_{\nu}[\rho]$ are nonincreasing right-continuous functions in $\nu$. Under the assumption of adiabaticity, the two integrands become equal to the derivatives $E_{\nu}^{\prime}[v]$ and $F_{\nu}^{\prime}[\rho]$ in Eqs. (11) and (12), respectively.

\section{Coulomb, exchange, and correlation energies}

It is customary to decompose the total interaction energies in Eqs. (13) and (14) in the manner

$$
\begin{aligned}
& \int_{0}^{\lambda} \mathcal{W}_{\nu}[v] \mathrm{d} \nu=J_{\lambda}[v]+E_{\mathrm{x}, \lambda}[v]+E_{\mathrm{c}, \lambda}[v], \\
& \int_{0}^{\lambda} \mathcal{W}_{\nu}[\rho] \mathrm{d} \nu=J_{\lambda}[\rho]+E_{\mathrm{x}, \lambda}[\rho]+E_{\mathrm{c}, \lambda}[\rho],
\end{aligned}
$$

where we have introduced the classical Coulomb functionals $\left(\rho_{0}^{v}\right.$ is the density associated with $\hat{\gamma}_{0}^{v}$ )

$$
\begin{aligned}
& J_{\lambda}[v]=\frac{1}{2} \iint w_{\lambda}\left(r_{12}\right) \rho_{0}^{v}\left(\mathbf{r}_{1}\right) \rho_{0}^{v}\left(\mathbf{r}_{2}\right) \mathrm{d} \mathbf{r}_{1} \mathrm{~d} \mathbf{r}_{2}, \\
& J_{\lambda}[\rho]=\frac{1}{2} \iint w_{\lambda}\left(r_{12}\right) \rho\left(\mathbf{r}_{1}\right) \rho\left(\mathbf{r}_{2}\right) \mathrm{d} \mathbf{r}_{1} \mathrm{~d} \mathbf{r}_{2},
\end{aligned}
$$

the exchange functionals

$$
\begin{aligned}
& E_{\mathrm{x}, \lambda}[v]=\operatorname{Tr} \hat{W}_{\lambda} \hat{\gamma}_{0}^{v}-J_{\lambda}[v], \\
& E_{\mathrm{x}, \lambda}[\rho]=\operatorname{Tr} \hat{W}_{\lambda} \hat{\gamma}_{0}^{\rho}-J_{\lambda}[\rho],
\end{aligned}
$$

and the correlation functionals 


$$
\begin{aligned}
& E_{\mathrm{c}, \lambda}[v]=\int_{0}^{\lambda} \mathcal{W}_{\mathrm{c}, \nu}[v] \mathrm{d} \nu, \quad \mathcal{W}_{\mathrm{c}, \nu}[v]=\operatorname{Tr} \hat{W}_{\nu}^{\prime}\left(\hat{\gamma}_{\nu}^{\nu}-\hat{\gamma}_{0}^{v}\right), \\
& E_{\mathrm{c}, \lambda}[\rho]=\int_{0}^{\lambda} \mathcal{W}_{\mathrm{c}, \nu}[\rho] \mathrm{d} \nu, \quad \mathcal{W}_{\mathrm{c}, \nu}[\rho]=\operatorname{Tr} \hat{W}_{\nu}^{\prime}\left(\hat{\gamma}_{\nu}^{\rho}-\hat{\gamma}_{0}^{\rho}\right) .
\end{aligned}
$$

To show Eqs. (21) and (22), we substitute Eqs. (23)-(28) in these equations and use the relation $\int_{0}^{\lambda} \hat{W}_{\nu}^{\prime} \mathrm{d} \nu=\hat{W}_{\lambda}$. The exchange and correlation energies may be combined to give the exchange-correlation energies, which by combination of Eqs. (25) and (26) with Eqs. (27) and (28) are given by

$$
\begin{aligned}
& E_{\mathrm{xc}, \lambda}[v]=\int_{0}^{\lambda} \mathcal{W}_{\mathrm{xc}, \nu}[v] \mathrm{d} \nu, \quad \mathcal{W}_{\mathrm{xc}, \nu}[v]=\operatorname{Tr} \hat{W}_{\nu}^{\prime} \hat{\gamma}_{\nu}^{\nu}-J_{\nu}^{\prime}[v], \\
& E_{\mathrm{xc}, \lambda}[\rho]=\int_{0}^{\lambda} \mathcal{W}_{\mathrm{xc}, \nu}[\rho] \mathrm{d} \nu, \quad \mathcal{W}_{\mathrm{xc}, \nu}[\rho]=\operatorname{Tr} \hat{W}_{\nu}^{\prime} \hat{\gamma}_{\nu}^{\rho}-J_{\nu}^{\prime}[\rho] .
\end{aligned}
$$

In the following, we shall study the potential- and densityfixed AC connections and their contributions for the helium isoelectronic series and $\mathrm{H}_{2}$ at different internuclear separations, with different choices of $\hat{W}_{\lambda}$.

\section{One- and two-electron contributions}

The Hamiltonian in Eq. (1) provides a natural decomposition of the total electronic energy into one- and twoelectron contributions,

$$
\begin{aligned}
& E_{\lambda}[v]=\operatorname{Tr} \hat{H}_{0}[v] \hat{\gamma}_{\lambda}^{v}+\operatorname{Tr} \hat{W}_{\lambda} \hat{\gamma}_{\lambda}^{v}, \\
& F_{\lambda}[\rho]=\operatorname{Tr} \hat{H}_{0}[0] \hat{\gamma}_{\lambda}^{\rho}+\operatorname{Tr} \hat{W}_{\lambda} \hat{\gamma}_{\lambda}^{\rho},
\end{aligned}
$$

which may be further decomposed into uncorrelated and correlated parts. The uncorrelated energy is obtained by the substitution $\hat{\gamma}_{\lambda}^{v} \rightarrow \hat{\gamma}_{0}^{v}$ in Eq. (31) and the substitution $\hat{\gamma}_{\lambda}^{\rho} \rightarrow \hat{\gamma}_{0}^{\rho}$ in Eq. (32),

$$
E_{\mathrm{u}, \lambda}[v]=\operatorname{Tr} \hat{H}_{0}[v] \hat{\gamma}_{0}^{v}+\operatorname{Tr} \hat{W}_{\lambda} \hat{\gamma}_{0}^{v}=H_{\mathrm{s}}[v]+J_{\lambda}[v]+E_{\mathrm{x}, \lambda}[v],
$$

$$
F_{\mathrm{u}, \lambda}[\rho]=\operatorname{Tr} \hat{H}_{0}[0] \hat{\gamma}_{0}^{\rho}+\operatorname{Tr} \hat{W}_{\lambda} \hat{\gamma}_{0}^{\rho}=T_{\mathrm{s}}[\rho]+J_{\lambda}[\rho]+E_{\mathrm{x}, \lambda}[\rho]
$$

The uncorrelated one-electron energies are thus simply the noninteracting energies $H_{\mathrm{s}}[v]$ and $T_{\mathrm{s}}[\rho]$ in Eqs. (15) and (16), respectively, whereas the uncorrelated two-electron energies are the Coulomb and exchange energies evaluated from $\hat{\gamma}_{0}^{v}$ and $\hat{\gamma}_{0}^{\rho}$, respectively. The correlation energy is next obtained by the substitutions $\hat{\gamma}_{\lambda}^{v} \rightarrow \hat{\gamma}_{\lambda}^{v}-\hat{\gamma}_{0}^{v}$ in Eq. (31) and $\hat{\gamma}_{\lambda}^{\rho} \rightarrow \hat{\gamma}_{\lambda}^{\rho}-\hat{\gamma}_{0}^{\rho}$ in Eq. (32), yielding

$$
\begin{aligned}
E_{\mathrm{c}, \lambda}[v] & =\operatorname{Tr} \hat{H}_{0}[v]\left(\hat{\gamma}_{\lambda}^{v}-\hat{\gamma}_{0}^{v}\right)+\operatorname{Tr} \hat{W}_{\lambda}\left(\hat{\gamma}_{\lambda}^{v}-\hat{\gamma}_{0}^{v}\right) \\
& =H_{\mathrm{c}, \lambda}[v]+E_{\mathrm{c}, \lambda}^{2 \mathrm{el}}[v], \\
E_{\mathrm{c}, \lambda}[\rho] & =\operatorname{Tr} \hat{H}_{0}[0]\left(\hat{\gamma}_{\lambda}^{\rho}-\hat{\gamma}_{0}^{\rho}\right)+\operatorname{Tr} \hat{W}_{\lambda}\left(\hat{\gamma}_{\lambda}^{\rho}-\hat{\gamma}_{0}^{\rho}\right) \\
& =T_{\mathrm{c}, \lambda}[\rho]+E_{\mathrm{c}, \lambda}^{2 \mathrm{el}}[\rho],
\end{aligned}
$$

where we use the conventional notation $E_{\mathrm{c}, \lambda}[\rho]=F_{\mathrm{c}, \lambda}[\rho]$. For the standard connection, $w_{\lambda}\left(r_{i j}\right)=\lambda / r_{i j}$ and it follows that $\hat{W}_{1}^{\prime}=\hat{W}_{1}$. Comparing the integrands in Eqs. (27) and (28) with the two-electron parts in Eqs. (35) and (36), we then find that the two-electron correlation energy is equal to the AC integrand at $\lambda=1: E_{\mathrm{c}, 1}^{2 \mathrm{el}}[v]=\mathcal{W}_{\mathrm{c}, 1}[v]$ and $E_{\mathrm{c}, 1}^{2 \mathrm{el}}[\rho]=\mathcal{W}_{\mathrm{c}, 1}[\rho]$. However, these relations are not valid for all possible connections $w_{\lambda}$.

Finally, we note that the one-electron contributions (kinetic and interaction with the external potential) to the total energy must be the same for conjugate $v$ and $\rho$,

$$
\begin{aligned}
H_{\mathrm{s}}[v]+H_{\mathrm{c}, \lambda}[v]= & T_{\mathrm{s}}[\rho]+T_{\mathrm{c}, \lambda}[\rho]+(v \mid \rho) \\
& (v \text { and } \rho \text { conjugate at } \lambda) .
\end{aligned}
$$

Combining this result with Fenchel's inequality for the noninteracting system Eq. (19), we obtain the inequality

$$
H_{\mathrm{c}, \lambda}[v] \geq T_{\mathrm{c}, \lambda}[\rho] \geq 0 \quad(v \text { and } \rho \text { conjugate at } \lambda),
$$

where the non-negativity of $T_{\mathrm{c}, \lambda}[\rho]$ follows from the definition of $T_{\mathrm{s}, \lambda}[\rho]$ as the lowest kinetic-energy expectation value consistent with the density $\rho$.

\section{E. Range-independent and range-dependent connections}

Thus far, we have established the potential-fixed integrands $\mathcal{W}_{\lambda}[v], \mathcal{W}_{\mathrm{xc}, \lambda}[v]$, and $\mathcal{W}_{\mathrm{c}, \lambda}[v]$ and density-fixed integrands $\mathcal{W}_{\lambda}[\rho], \mathcal{W}_{\mathrm{xc}, \lambda}[\rho]$, and $\mathcal{W}_{\mathrm{c}, \lambda}[\rho]$, whose couplingconstant integration yields the total interaction energy, the exchange-correlation energy, and the correlation energy, respectively. Yang ${ }^{27}$ observed that since these integrals are determined entirely by the functional values at the end points of the integration $\left(\lambda_{2}>\lambda_{1}\right)$,

$$
\begin{aligned}
& \int_{\lambda_{1}}^{\lambda_{2}} \mathcal{W}_{\lambda}[v] \mathrm{d} \lambda=E_{\lambda_{2}}[v]-E_{\lambda_{1}}[v], \\
& \int_{\lambda_{1}}^{\lambda_{2}} \mathcal{W}_{\lambda}[\rho] \mathrm{d} \lambda=F_{\lambda_{2}}[\rho]-F_{\lambda_{1}}[\rho],
\end{aligned}
$$

we may choose $\hat{W}_{\lambda}$ freely in Eq. (1) provided its end-point values (typically 0 and 1) are unaffected. This idea provides a justification for the proposal of $\operatorname{Savin}^{28}$ to construct a variety of hybrid theories that merge wave-function approaches with DFT from the viewpoint of a generalized AC. ${ }^{27}$ For further discussion see Sec. III E.

While some studies have appeared examining the integrated quantities $E_{\mathrm{xc}}[\rho]$ and $E_{\mathrm{c}}[\rho]$ for the density-fixed connection, ${ }^{21,50}$ no explicit study of the integrands involved, varying the path between the noninteracting and interacting 
systems, has been carried out. In the present work, we consider the following general forms for $w_{\lambda}\left(r_{i j}\right)$ in Eq. (3):

$$
\begin{aligned}
w_{\lambda}^{\mathrm{s}}\left(r_{i j}\right)= & \frac{\lambda}{r_{i j}} \quad \text { (standard), } \\
w_{\lambda}^{\mathrm{e}}\left(r_{i j}\right)= & \frac{\operatorname{erf}\left(\frac{\lambda}{1-\lambda} r_{i j}\right)}{r_{i j}} \quad \text { (error function) } \\
w_{\lambda}^{\mathrm{g}}\left(r_{i j}\right)= & \frac{\operatorname{erf}\left(\frac{\lambda}{1-\lambda} r_{i j}\right)}{r_{i j}}-\frac{2}{\sqrt{\pi}}\left(\frac{\lambda}{1-\lambda}\right) \\
& \times \exp \left(-\frac{1}{3}\left(\frac{\lambda}{1-\lambda}\right)^{2} r_{i j}^{2}\right)
\end{aligned}
$$

whose $\lambda$ derivatives are given by

$$
\begin{aligned}
w_{\lambda}^{\mathrm{s}^{\prime}}\left(r_{i j}\right)= & \frac{1}{r_{i j}}, \\
w_{\lambda}^{\mathrm{e}^{\prime}}\left(r_{i j}\right)= & \frac{2 \exp \left(-\left(\frac{\lambda}{1-\lambda}\right)^{2} r_{i j}^{2}\right)}{\sqrt{\pi}(1-\lambda)^{2}}, \\
w_{\lambda}^{\mathrm{g}^{\prime}}\left(r_{i j}\right)= & \frac{2 \exp \left(-\left(\frac{\lambda}{1-\lambda}\right)^{2} r_{i j}^{2}\right)}{\sqrt{\pi}(1-\lambda)^{2}} \\
& +\frac{2\left(\frac{2}{3}\left(\frac{\lambda}{1-\lambda}\right)^{2} r_{i j}^{2}-1\right) \exp \left(-\frac{1}{3}\left(\frac{\lambda}{1-\lambda}\right)^{2} r_{i j}^{2}\right)}{\sqrt{\pi}(1-\lambda)^{2}} .
\end{aligned}
$$

The choice $w_{\lambda}^{\mathrm{s}}$ in Eq. (41) represents the standard rangeindependent $\mathrm{AC}$, depending linearly on $\lambda$. As $\lambda$ increases, the interaction is turned on uniformly for all interelectronic separations $r_{i j}$. By contrast, with the error-function connection $w_{\lambda}^{\mathrm{e}}$ in Eq. (42) and Gaussian-attenuated error-function connection $w_{\lambda}^{\mathrm{g}}$ in Eq. (43), the interaction is turned on in a rangedependent, nonuniform manner by the use of the functions $\operatorname{erf}\left(\mu r_{i j}\right)$, and $\exp \left(-\mu^{2} r_{i j}^{2} / 3\right)$ of $r_{i j}$, where $\mu=\lambda /(1-\lambda)$ varies over the range $0 \leq \mu \leq \infty$ when $\lambda$ increases from 0 to 1 . As a result, with these two connections, long-range interactions are accounted for first and short-range interactions last. To illustrate the difference between the above connections, we have in Fig. 1 plotted the functions in Eqs. (41)-(43) and their derivatives in Eqs. (44)-(46) as functions of $r_{i j}$, for four different values of $\lambda$. The $\lambda$ dependence of the derivatives is relevant since in the evaluation of the AC integrands in Eqs. (17) and (18), we calculate the expectation value of the density matrix with these derivatives.

To evaluate the AC integrands corresponding to the different choices of $\hat{W}_{\lambda}$ in Eqs. (41)-(43), we must calculate the expectation values of $\hat{W}_{\lambda}^{\prime}$ with a wave function corresponding to a fixed potential or a fixed density, determined by the
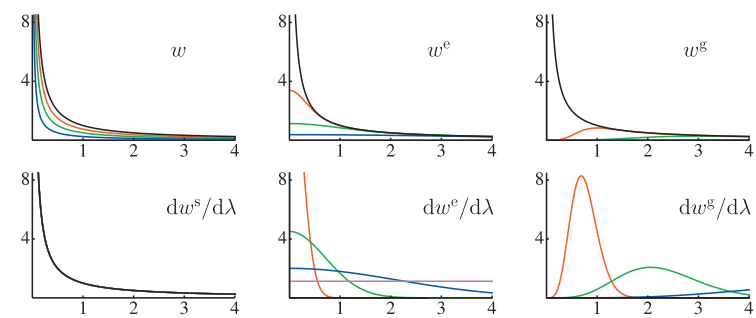

FIG. 1. Attenuated operators (top row) and their $\lambda$ derivatives (bottom row) as functions of $r_{12}$ for $\lambda=0$ (pink line), $\lambda=1 / 4$ (blue line), $\lambda=1 / 2$ (green line), $\lambda=3 / 4$ (red line), and $\lambda=1$ (black line).

optimizations of Eqs. (6) and (7). The minimization of Eq. (6) requires only standard techniques with two-electron integrals modified as described, for example, in Ref. 57. The maximization of Eq. (7) is more difficult but can be achieved quite efficiently by the method in Refs. 22 and 23. Expectation values of the derivatives in Eqs. (44)-(46) with the optimized wave functions necessary for the calculation of the generalized AC integrands require the evaluation of twoelectron integrals of the types $\left(a b\left|\exp \left(-\gamma r_{i j}^{2}\right)\right| c d\right)$ and $\left(a b\left|r_{i j}^{2} \exp \left(-\gamma r_{i j}^{2}\right)\right| c d\right)$, where the exponent $\gamma$ is determined by $\lambda$. Such integrals occur in R12 theories and as such are available in a variety of codes; in the present work, we use the integrals implemented for R12 theories by Samson et al. ${ }^{58}$ specifically the $I_{2}$ and $I_{4}$ integrals of that paper. The procedure is then to choose a suitable wave function for accurate determination of the Lieb functional, as described in Refs. 22 and 23 using modified two-electron integrals according to the choice of two-electron interaction from Eqs. (41)-(43). Once optimized, the expectation values of the derivatives required for the calculation of the $\mathrm{AC}$ integrands in Eqs. (44)-(46) are calculated, the linear AC being particularly simple since only the standard expectation value of the usual two-electron operator is required, see Eq. (44).

\section{RESULTS}

\section{A. Computational details}

The Lieb maximization of Eq. (7) has been performed using the algorithm proposed by Wu and Yang ${ }^{23}$ (see Ref. 18 for an alternative approach), which has been implemented recently ${ }^{22}$ in the DALTON quantum chemistry $\operatorname{program}^{59}$ for arbitrary interaction strengths and the generalized ACs discussed in Sec. II E. The reader is referred to Refs. 22 and 23 for details of the implementation; here we note that the key to the approach of $\mathrm{Wu}$ and Yang ${ }^{23}$ to perform the Lieb maximization is the parametrization of the potential in the manner,

$$
v_{\mathbf{b}}(\mathbf{r})=v_{\mathrm{ext}}(\mathbf{r})+v_{\mathrm{ref}, \lambda}(\mathbf{r})+\sum_{t} b_{t} g_{t}(\mathbf{r}),
$$

where the first term $v_{\text {ext }}(\mathbf{r})$ is the external potential due to the nuclei, the second term $v_{\text {ref }, \lambda}(\mathbf{r})$ is a fixed reference potential chosen to ensure the correct asymptotic behavior, and the final term is a linear expansion in Gaussian functions $g_{t}(\mathbf{r})$ with coefficients $b_{t}$. Inserting this expansion into Eq. (7) and using the gradient and Hessian with respect to the coefficients $b_{t}$, the Lieb maximization may be performed using 
standard quasi-Newton or Newton techniques. Here we have used the Newton method employing both the gradient and Hessian with a truncated singular-value decomposition cutoff of $10^{-6}$ and a convergence target of less than $10^{-6}$ on the gradient norm; for further details see Refs. 22 and 23. All of the energies $E_{\lambda}[v]$ for the two-electron systems in the present work are calculated at the FCI level.

\section{B. The choice of basis sets}

In order to perform the Lieb maximization we therefore must choose both a primary orbital basis set and an auxiliary potential basis set. When the potential basis set is chosen to be very different to that of the orbital basis set, unphysical oscillatory potentials can be obtained. This problem has been widely discussed in the literature in the context of the optimized effective potential method ${ }^{60-71}$ and more recently in the context of constrained-search procedures ${ }^{72}$ at $\lambda=0$. To illustrate these effects we examine the exchange-correlation potentials for the helium atom along the density-fixed rangeindependent $\mathrm{AC}$ in Fig. 2. The uncontracted aug-cc-pVXZ basis sets have been employed for both the orbital and potential expansions. The potentials plotted in Fig. 2 represent the combinations $X=\{6,2\}, X=\{4,4\}$, and $X=\{2,6\}$, where the first number refers to the orbital-basis cardinal number, $X_{\text {orb }}$, and the second to the potential-basis cardinal number, $X_{\text {pot }}$. For each combination, the potentials for interaction strengths $\lambda$ from 0.0 to 1.0 are shown in increments of 0.1 .

The combination $X=\{6,2\}$ may be regarded as unbalanced in the sense that the potential basis is much smaller than that of the orbital-basis; while the potentials for this combination are smooth, the lack of flexibility in the potential expansion may limit the variational freedom of the calculation. The combination $X=\{4,4\}$ represents a balanced choice; here a small peak can been seen close to the nucleus, although the potential is predominantly smooth. It can be removed by application of the smoothing norm procedure of Ref. 72 as was done in our previous work. ${ }^{22}$ The final combination $X=\{2,6\}$ is unbalanced in the sense that the potential basis set is much larger than the orbital basis set; for this combination the unphysical feature at the nucleus becomes much larger. Further increasing the size of the potential basis set can cause these oscillations to grow further.

In Table I we explore the impact of different choices for the auxiliary potential basis set on the expectation value $\mathcal{W}_{\lambda}[\rho]$, which is central to the calculation of the densityfixed $\mathrm{AC}$ in Eq. (14). Results are presented for $\lambda=0.0,0.5$, and 1.0. Each row represents a choice of orbital basis, with each column corresponding to a different potential basis. For each value of $\lambda$ and all choices of orbital basis, it is apparent that the expectation value is remarkably stable with respect to variations in the auxiliary basis. Furthermore, these small variations are largest for $\lambda=0$ where the potential is largest (see Fig. 2) and reduce steadily to zero for $\lambda=1.0$, where the potential is zero.

The expectation value has much more significant variation with respect to the orbital basis cardinal number, as would be expected. For each value of $\lambda$, we present $\mathcal{W}_{\lambda}[\rho]$

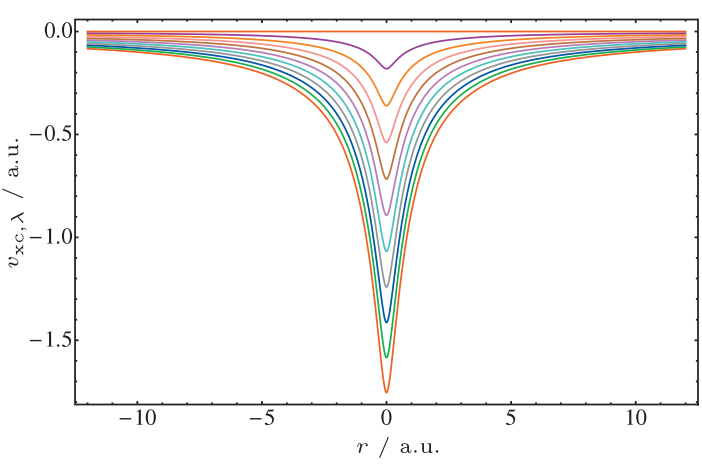

(a)

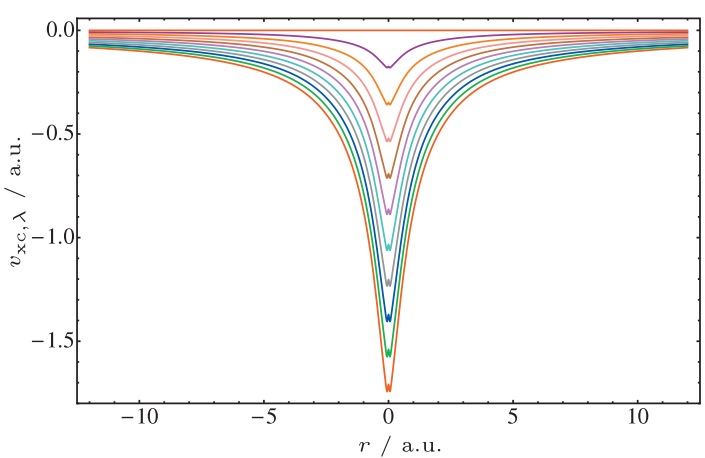

(b)

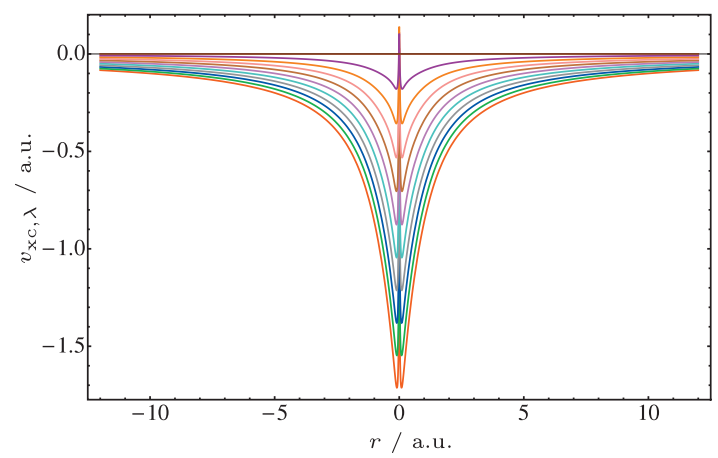

(c)

FIG. 2. Exchange-correlation potentials for the helium atom along the density-fixed AC with $\lambda=0.0-1.0$ in steps of 0.1 . Pane (a) corresponds to the basis set combination $X=\{6,2\}$ for the orbital basis and potential basis cardinal numbers, respectively. Pane (b) corresponds to the combination $X=\{4,4\}$ and pane (c) to the combination $X=\{6,2\}$.

for orbital basis sets with $2 \leq X_{\text {orb }} \leq 6$, along with an estimate of the basis-set-limit value calculated using the two-point extrapolation formula

$$
E_{X Y}=\frac{X^{3} E_{X}-Y^{3} E_{Y}}{X^{3}-Y^{3}}
$$

in Ref. 73 with $X=5$ and $Y=6$. These extrapolated results are denoted [56]; for a discussion of the application of this formula in the context of AC calculations, see Ref. 22. In the final row for each value of $\lambda$ we have presented the deviation of the $X_{\text {orb }}=4$ values from the estimated orbital basis set limit, denoted by $\Delta$. For all interaction strengths between 0 and 1 the absolute deviation from this limit is less than 1 mhartree. In light of this analysis we choose the uncontracted aug-cc-p(C)VQZ basis sets for both the orbital and potential 
TABLE I. The variation of the expectation value $\mathcal{W}_{\lambda}[\rho]$ with choice of orbital and auxiliary potential expansion basis sets for the density fixed AC of the helium atom. The uncontracted aug-cc-pVXZ basis sets have been used for both expansions. Each row represents the change in the expectation value with for a given orbital basis cardinal number $X_{\mathrm{orb}}$ as the cardinal number of the potential basis, $X_{\text {pot }}$, is changed. For the definition of the quantities [56] and $\Delta$ see text. Exchange-correlation potentials corresponding to the values marked in bold are shown in Fig. 2. All values in atomic units.

\begin{tabular}{|c|c|c|c|c|c|}
\hline Orbital basis $X_{\text {orb }}$ & 2 & 3 & 4 & 5 & 6 \\
\hline 2 & 1.017678 & 1.017700 & 1.017709 & 1.017707 & 1.017707 \\
\hline 3 & 1.023222 & 1.023224 & 1.023232 & 1.023229 & 1.023230 \\
\hline 4 & 1.024084 & 1.024084 & 1.024084 & 1.024086 & 1.024085 \\
\hline$[56]$ & 1.024628 & 1.024628 & 1.024628 & 1.024628 & 1.024628 \\
\hline$\Delta=\mathcal{W}_{\lambda}^{4}-\mathcal{W}_{\lambda}^{[56]}$ & -0.000544 & -0.000544 & -0.000543 & -0.000542 & -0.000543 \\
\hline \multicolumn{6}{|c|}{$\lambda=0.5$} \\
\hline 2 & 0.981488 & 0.981504 & 0.981511 & 0.981509 & 0.981510 \\
\hline [56] & 0.981155 & 0.981155 & 0.981155 & 0.981155 & 0.981155 \\
\hline$\Delta=\mathcal{W}_{\lambda}^{4}-\mathcal{W}_{\lambda}^{[56]}$ & 0.000436 & 0.000430 & 0.000436 & 0.000437 & 0.000436 \\
\hline \multicolumn{6}{|c|}{$\lambda=1.0$} \\
\hline 2 & 0.949929 & 0.949929 & 0.949929 & 0.949929 & 0.949929 \\
\hline 3 & 0.947658 & 0.947658 & 0.947658 & 0.947658 & 0.947658 \\
\hline 4 & 0.946580 & 0.946580 & 0.946580 & 0.946580 & 0.946580 \\
\hline 5 & 0.946225 & 0.946225 & 0.946225 & 0.946225 & 0.946225 \\
\hline 6 & 0.946065 & 0.946065 & 0.946065 & 0.946065 & 0.946065 \\
\hline$[56]$ & 0.945844 & 0.945844 & 0.945844 & 0.945844 & 0.945844 \\
\hline$\Delta=\mathcal{W}_{\lambda}^{4}-\mathcal{W}_{\lambda}^{[56]}$ & 0.000735 & 0.000735 & 0.000735 & 0.000735 & 0.000735 \\
\hline
\end{tabular}

expansions for all of the two-electron systems in this study and quote all energetic values to a precision of 1 mhartree. This choice of basis represents a good compromise between computational efficiency, accuracy, and adequate representation of the exchange-correlation potential. Finally, we note that for the potential-fixed AC when calculating the expectation value $\mathcal{W}_{\lambda}[v]$, we fix the potential $v$ at the physical external potential (due to the nuclei) for all values of the interaction strength. As such, only the orbital basis set plays a role in these calculations and similar accuracy is achieved in the uncontracted aug-cc-p $(\mathrm{C}) \mathrm{VQZ}$ basis sets.

\section{The helium isoelectronic series}

The helium isoelectronic series has been extensively studied and poses a significant challenge for approximate exchange-correlation functionals, particularly as $Z$ increases. ${ }^{17,18,74-78}$ In the present work, we examine the systems with $1 \leq Z \leq 10$ using the uncontracted aug-cc-pCVQZ basis set, ${ }^{79-82}$ noting that uncontraction and the use of corecorrelating functions are essential to describe the compact densities accurately. The total energy and its components are listed in Table II, for the density-fixed connection (columns 3-9) and the potential-fixed connection (columns 10-12). In Fig. 3, we have plotted the total AC integrands $\mathcal{W}_{\lambda}[v]$ and $\mathcal{W}_{\lambda}[\rho]$ for the three connections $w_{\lambda}^{\mathrm{s}}, w_{\lambda}^{\mathrm{e}}$, and $w_{\lambda}^{\mathrm{g}}$ in Eqs. (41)-(43), respectively.
As $Z$ increases in the isoelectronic series, the density becomes more compact and may, to a good approximation, be expressed by a scaling of the density in $\mathrm{H}^{-}: \rho_{Z}(\mathbf{r})$ $\approx Z^{3} \rho_{\mathrm{H}^{-}}(Z \mathbf{r})$. Consequently, the energy and its components increase in magnitude with increasing $Z$, in an approximately linear manner-see Table II. The only exception to this behavior are the correlation energies, which remain approximately constant with $Z$. These observations are in agreement with well-known scaling relations, such as $J_{\lambda}\left[\rho_{Z}\right]=Z J_{\lambda}\left[\rho_{\mathrm{H}^{-}}\right]$ for the classical Coulomb energy.

Concerning the quality of the one-electron basis set, we note that the calculated bare-nucleus energy $H_{\mathrm{s}}[v]$ in Table II differs from the exact bare-nucleus energy $H_{\mathrm{s}}[v]=Z^{2}$ by less than $0.001 E_{\mathrm{h}}$. Moreover, the virial theorem is satisfied to better than $1 \%$ for $\mathrm{H}^{-}$and better than $0.1 \%$ for the remaining systems.

Comparing the density- and potential-fixed results in Table II, we first note that $H_{\mathrm{s}}[v]$ is lower than $T_{\mathrm{s}}[\rho]+(v \mid \rho)$ by $13 \%$ for $Z=1$, by $3 \%$ for $Z=2$, and by $0.1 \%$ for $Z=10$, in agreement with Eq. (19). Likewise, the positive quantity $H_{\mathrm{c}}[v]$ is several times larger than $T_{\mathrm{c}}[\rho]$ for all $Z$, in agreement with Eq. (38). Finally, comparing the positive quantities $\int \mathcal{W}_{\lambda}[v] \mathrm{d} \lambda \geq \int \mathcal{W}_{\lambda}[\rho] \mathrm{d} \lambda$ [see Eq. (20)], we find that the former is larger than the latter by $27 \%$ for $Z=1$, by $10 \%$ for $Z=2$, and by $2 \%$ for $Z=10$. As expected, the energy changes that occur in the potential-fixed system with increasing $\lambda$ are 
TABLE II. Energy components of the helium isoelectronic series in the uncontracted aug-cc-pCVQZ basis (atomic units).

\begin{tabular}{|c|c|c|c|c|c|c|c|c|c|c|c|}
\hline Z & $E_{\mathrm{tot}}(Z)$ & $T_{\mathrm{s}}[\rho]$ & $(v \mid \rho)$ & $\int \mathcal{W}_{\lambda}[\rho] \mathrm{d} \lambda$ & $J[\rho]$ & $E_{\mathrm{x}}[\rho]$ & $E_{\mathrm{c}}[\rho]$ & $T_{\mathrm{c}}[\rho]$ & $H_{\mathrm{s}}[v]$ & $\int \mathcal{W}_{\lambda}[v] \mathrm{d} \lambda$ & $H_{\mathrm{c}}[v]$ \\
\hline 1 & -0.527 & 0.502 & -1.374 & 0.345 & 0.773 & -0.386 & -0.041 & 0.028 & -1.000 & 0.473 & 0.155 \\
\hline 2 & -2.903 & 2.865 & -6.751 & 0.983 & 2.048 & -1.024 & -0.041 & 0.036 & -4.000 & 1.097 & 0.150 \\
\hline 3 & -7.279 & 7.238 & -16.126 & 1.609 & 3.302 & -1.651 & -0.042 & 0.039 & -9.000 & 1.721 & 0.152 \\
\hline 4 & -13.654 & 13.613 & -29.501 & 2.234 & 4.553 & -2.277 & -0.043 & 0.041 & -16.000 & 2.346 & 0.153 \\
\hline 5 & -22.030 & 21.987 & -46.875 & 2.858 & 5.804 & -2.902 & -0.043 & 0.041 & -25.000 & 2.970 & 0.153 \\
\hline 6 & -32.405 & 32.361 & -68.249 & 3.483 & 7.054 & -3.527 & -0.044 & 0.042 & -36.000 & 3.595 & 0.154 \\
\hline 7 & -44.780 & 44.735 & -93.623 & 4.108 & 8.304 & -4.152 & -0.044 & 0.042 & -49.000 & 4.220 & 0.154 \\
\hline 8 & -59.155 & 59.109 & -122.998 & 4.733 & 9.554 & -4.777 & -0.044 & 0.043 & -64.000 & 4.845 & 0.154 \\
\hline 9 & -75.530 & 75.484 & -156.372 & 5.359 & 10.805 & -5.402 & -0.044 & 0.043 & -81.000 & 5.470 & 0.154 \\
\hline 10 & -93.905 & 93.859 & -193.747 & 5.984 & 12.055 & -6.027 & -0.044 & 0.043 & -100.000 & 6.095 & 0.154 \\
\hline
\end{tabular}

larger in the corresponding density-fixed system. Physically, these relations may be understood from the observation that the density of the potential-fixed noninteracting system is more compact than that of the density-fixed system. We also note that the differences between the potential- and densityfixed quantities are largest for $Z=1$, which has the most diffuse electron density. This behavior may be understood from the observation that in anionic systems, the diffuseness of the electron density arises from electron repulsion, which is neglected in the noninteracting limit, generating a too compact density in the potential-fixed connection.

A further comparison of the density- and potential-fixed ACs is given in Fig. 3, where we have plotted $\mathcal{W}_{\lambda}[v]$ and $\mathcal{W}_{\lambda}[\rho]$ against $\lambda$ for $1 \leq Z \leq 10$, where $w_{\lambda}^{\mathrm{s}}$ is in pane (a), $w_{\lambda}^{\mathrm{e}}$ in pane (b), and $w_{\lambda}^{\mathrm{g}}$ in pane (c). The density- and potentialfixed integrands are very similar and we note that for all values of $\lambda$ and all three connections, $\mathcal{W}_{\lambda}[v] \geq \mathcal{W}_{\lambda}[\rho]$, as may be rationalized by observing that the density becomes more compact in the potential-fixed $\mathrm{AC}$ as the electronic interactions are turned off.

Comparing the $\mathrm{AC}$ curves arising from the different choices of $w_{\lambda}$ in Fig. 3, we recall that each curve represents the expectation value of $\hat{W}_{\lambda}^{\prime}$ with $\hat{\gamma}_{\lambda}^{\rho}$ or $\hat{\gamma}_{\lambda}^{v}$, optimized with the two-electron operator $\hat{W}_{\lambda}$. The standard connection $w_{\lambda}^{\mathrm{s}}$ in pane (a) yields nearly straight lines, with a larger slope in the potential-fixed case (dashed lines) than in the density-fixed case (full lines), representing a situation where the interactions are turned on uniformly for all interelectronic separations. The $w_{\lambda}^{\mathrm{e}}$ curves in pane (b) give the same total interactions as those in pane (a) but have very different shapes since the interactions are now first turned on for large interelectronic separations and subsequently for short separations. The AC curves are therefore no longer linear but contain a peak at that value of $\lambda$ where most of the interactions are recovered. For $Z=1$, the peak is broad and occurs already at $\lambda \approx 0.1$, reflecting the large range of interelectronic separations that contribute to the interactions in this diffuse system. For the most compact system with $Z=10$, there is a sharp peak at $\lambda \approx 0.87$, indicating that most interactions occur at about $0.2 a_{0}-0.3 a_{0}$. The $w_{\lambda}^{\mathrm{g}}$ plots in pane (c) are similar to those in pane (b) but have shaper peaks, reflecting the higher locality of $w_{\lambda}^{\mathrm{g}^{\prime}}$, see Fig. 1 .

For the density-fixed AC, we consider separately also the

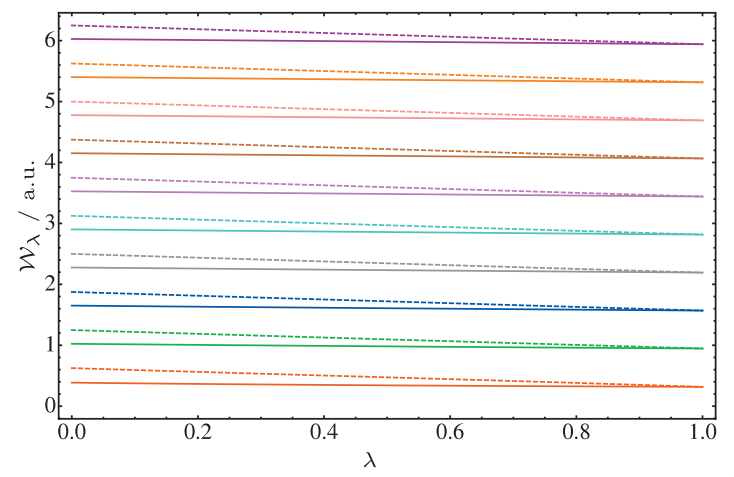

(a)

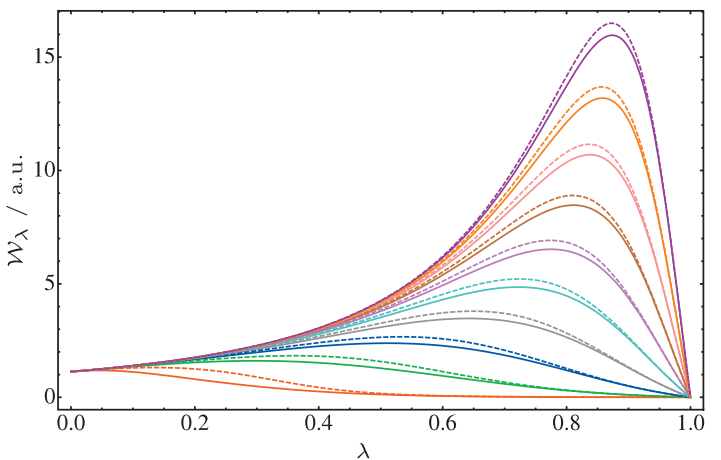

(b)

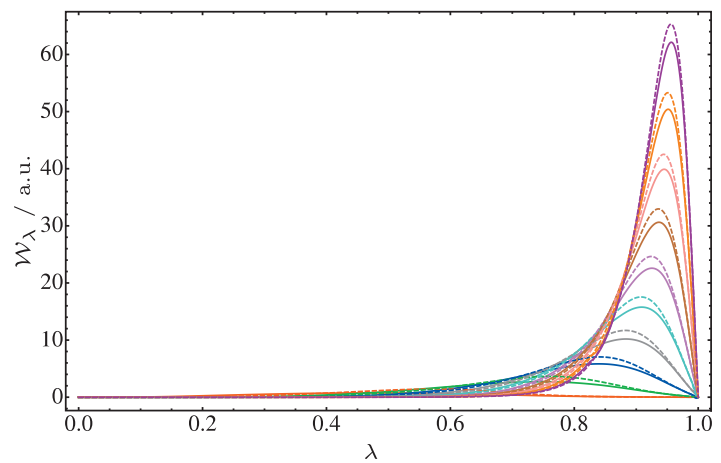

(c)

FIG. 3. AC curves (atomic units) $\mathcal{W}_{\lambda}[\rho]$ (full lines) and $\mathcal{W}_{\lambda}[v]$ (dashed lines) for the helium isoelectronic series with $1 \leq Z \leq 10$ for $w_{\lambda}^{\mathrm{s}}$ in pane (a), for $w_{\lambda}^{\mathrm{e}}$ in pane (b), and for $w_{\lambda}^{\mathrm{g}}$ in pane (c). In all panes, the curves increase with increasing $Z$. 


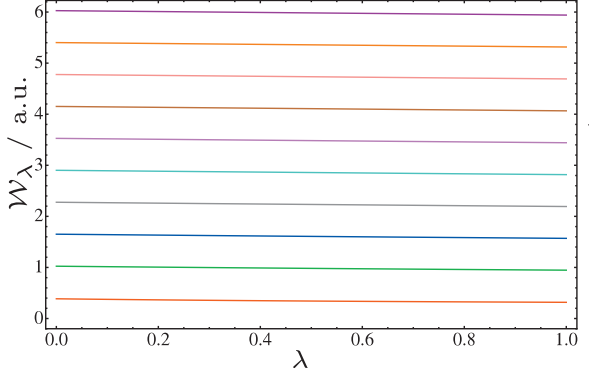

(a)

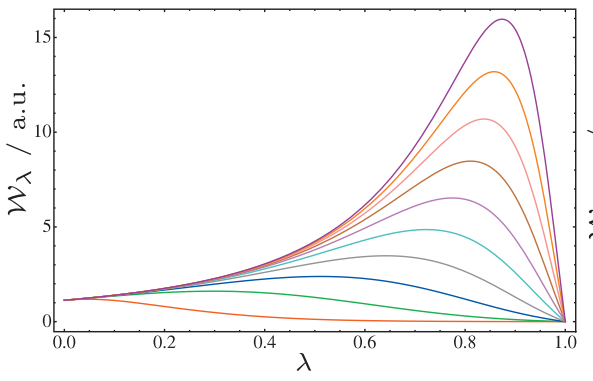

(d)

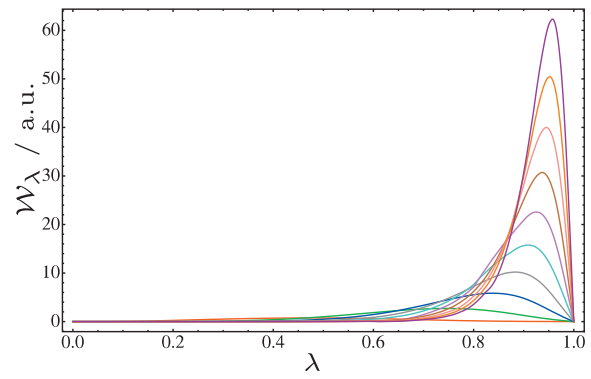

(g)

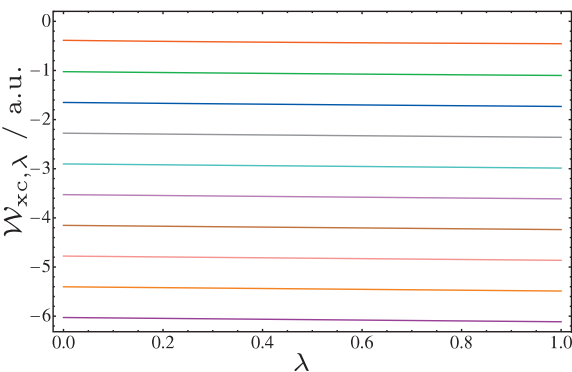

(b)

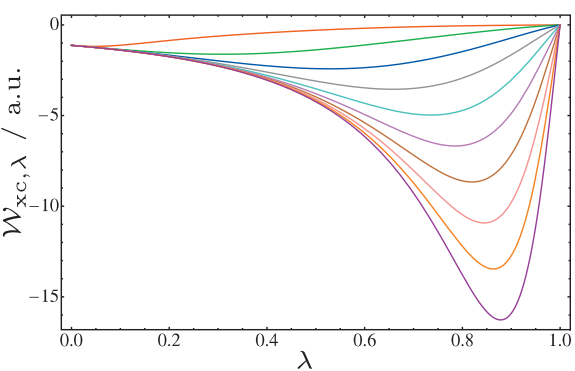

(e)

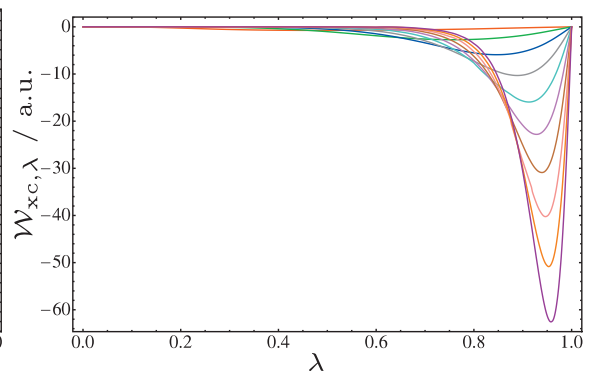

(h)

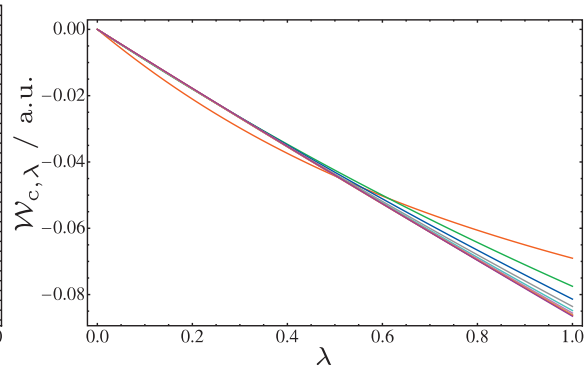

(c)

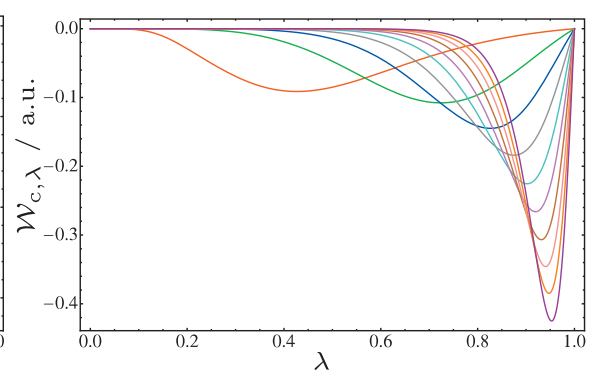

(f)

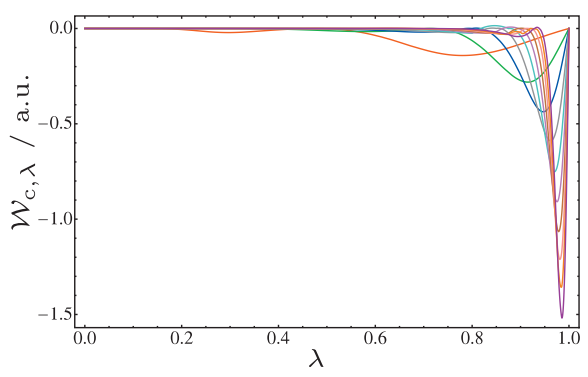

(i)

FIG. 4. AC curves (atomic units) for the helium isoelectronic series with $1 \leq Z \leq 10$ for $w_{\lambda}^{\mathrm{s}}$ in panes (a)-(c), for $w_{\lambda}^{\mathrm{e}}$ in panes (d)-(f), and for $w_{\lambda}^{\mathrm{g}}$ in panes (g)-(i). For each $\mathrm{AC}$, we have plotted the total curve $\mathcal{W}_{\lambda}[\rho]$ to the left, the exchange-correlation curve $\mathcal{W}_{\mathrm{xc}, \lambda}[\rho]$ in the middle, and the correlation curve $\mathcal{W}_{\mathrm{c}, \lambda}[\rho]$ to the right. In panes (a), (d), and (g), the curves increase with increasing $Z$; in the other panes, the curves may be distinguished by noting that the same color scheme is used in all panes.

exchange-correlation and correlation contributions to the full AC curve: $\mathcal{W}_{\lambda}[\rho]=\mathcal{W}_{\mathrm{xc}, \lambda}[\rho]+\mathcal{W}_{\mathrm{c}, \lambda}[\rho]$. In Fig. 4, we have plotted $\mathcal{W}_{\lambda}^{\mathrm{s}}[\rho], \mathcal{W}_{\lambda}^{\mathrm{e}}[\rho]$, and $\mathcal{W}_{\lambda}^{\mathrm{g}}[\rho]$ and their exchangecorrelation and correlation contributions for the helium isoelectronic series. The $\mathcal{W}_{\lambda}^{\mathrm{s}}[\rho]$ curves in pane (a) are positive and nearly constant since the dominant Coulomb and exchange energies increase linearly with $\lambda: \mathcal{W}_{\lambda}^{\mathrm{s}}[\rho] \approx J_{1}[\rho]$ $+E_{\mathrm{x}, 1}[\rho]=J_{1}[\rho] / 2$. The exchange-correlation curves in pane (b) are dominated by the exchange energy $\mathcal{W}_{\mathrm{xc}, \lambda}^{\mathrm{s}}[\rho]$ $\approx E_{\mathrm{x}, 1}[\rho]$ and are approximate mirror images of the curves in pane (a).

Pane (c) in Fig. 4 shows the correlation-only integrand $\mathcal{W}_{\mathrm{c}, \lambda}^{\mathrm{s}}[\rho]$ for the helium isoelectronic series, on a much larger scale than that used in panes (a) and (b). The curvature of the $\mathrm{H}^{-}$curve is much more pronounced than for the other species; as $Z$ increases, the density accumulates close to the nucleus and the curves become more linear. This behavior can be understood from the relation $E_{\mathrm{c}}\left[\rho_{Z}\right] \approx Z^{2} E_{\mathrm{c}, 1 / Z}\left[\rho_{\mathrm{H}^{-}}\right]$, which follows from the observed scaling of $\rho_{Z}$ with increasing $Z$ and a general scaling relation of the correlation energy ${ }^{83}$ For larger $Z$, the AC effectively explores a smaller $\lambda$ interval of some approximately universal AC curve valid for all Z. Consequently, these curves become more linear with increasing charge as the system approaches the highdensity limit. This trend toward linearity and the rate at which it occurs are clear in Fig. 4. We note that linearity of the correlation AC curve means that the correlation energy increases quadratically with $\lambda$, as expected from the validity of second-order Görling-Levy perturbation theory ${ }^{55,56}$ for these systems.

In the range-dependent error-function curves in the second row of Fig. 4, long-range interactions are recovered for small values of $\lambda$, while short-range interactions are recovered for large $\lambda$ values. For the total integrand $\mathcal{W}_{\lambda}^{\mathrm{e}}[\rho]$ in pane (d), the height of the peak increases and moves to the right with increasing $Z$, as the density contracts and the interactions become more short-ranged. As for the standard connection $w_{\lambda}^{\mathrm{s}}$, the exchange-correlation in pane (e) is an approximate mirror image of the total curve in pane (d): $\mathcal{W}_{\mathrm{xc}, \lambda}^{\mathrm{e}}[\rho]$ $\approx-\mathcal{W}_{\lambda}^{\mathrm{e}}[\rho]$. The range separation induced by the error func- 
TABLE III. Energy components of the $\mathrm{H}_{2}$ molecule in the aug-cc-pVQZ basis (atomic units).

\begin{tabular}{|c|c|c|c|c|c|c|c|c|c|c|c|}
\hline$R$ & $E_{\mathrm{tot}}(R)$ & $T_{\mathrm{s}}[\rho]$ & $(v \mid \rho)$ & $\int \mathcal{W}_{\lambda}[\rho] \mathrm{d} \lambda$ & $J[\rho]$ & $E_{\mathrm{x}}[\rho]$ & $E_{\mathrm{c}}[\rho]$ & $T_{\mathrm{c}}[\rho]$ & $H_{\mathrm{s}}[v]$ & $\int \mathcal{W}_{\lambda}[v] \mathrm{d} \lambda$ & $H_{\mathrm{c}}[v]$ \\
\hline 0.7 & -0.921 & 1.731 & -4.869 & 0.788 & 1.653 & -0.827 & -0.039 & 0.033 & -3.222 & 0.872 & 0.117 \\
\hline 1.4 & -1.174 & 1.141 & -3.650 & 0.621 & 1.323 & -0.661 & -0.041 & 0.033 & -2.568 & 0.680 & 0.093 \\
\hline 3.0 & -1.057 & 0.828 & -2.619 & 0.400 & 0.955 & -0.477 & -0.077 & 0.042 & -1.822 & 0.431 & 0.073 \\
\hline 5.0 & -1.004 & 0.953 & -2.382 & 0.226 & 0.820 & -0.410 & -0.184 & 0.022 & -1.449 & 0.245 & 0.042 \\
\hline 7.0 & -1.000 & 0.993 & -2.284 & 0.147 & 0.767 & -0.384 & -0.236 & 0.005 & -1.297 & 0.154 & 0.011 \\
\hline 10.0 & -1.000 & 1.000 & -2.199 & 0.100 & 0.725 & -0.362 & -0.262 & 0.000 & -1.201 & 0.101 & 0.001 \\
\hline
\end{tabular}

tion is particularly pronounced for the correlation curves $\mathcal{W}_{\mathrm{c}, \lambda}^{\mathrm{e}}[\rho]$ in pane (f), where the plotting scale is about 50 times larger than in panes (d) and (e).

To understand the behavior of the error-function curves at the end points, we note that $w_{0}^{\mathrm{e}^{\prime}}\left(r_{i j}\right)=2 / \sqrt{\pi}$, implying that $\operatorname{Tr} \hat{W}_{0}^{\prime} \hat{\gamma}=N(N-1) / \sqrt{\pi}$ and $J_{0}[\rho]=N^{2} / \sqrt{\pi}$. It follows that $\mathcal{W}_{0}^{\mathrm{e}}[\rho]=N(N-1) / \sqrt{\pi}, \mathcal{W}_{\mathrm{xc}, 0}^{\mathrm{e}}[\rho]=-N / \sqrt{\pi}$, and $\mathcal{W}_{\mathrm{c}, 0}^{\mathrm{e}}[\rho]=0$ for a noninteracting $N$-electron system. In the interacting limit, all integrands vanish since $w_{1}^{\mathrm{e}^{\prime}}\left(r_{i j}\right)=0$. We have thus established a way to transform the shape of the AC such that the noninteracting and interacting points are known constants. This behavior contrasts with the standard connection, in which the noninteracting point is a two-electron expectation value with the noninteracting wave function, whereas the interacting point is the expectation value of the two-electron operator with the fully interacting wave function.

The curves in the final row of Fig. 4 show the ACs for the Gaussian-attenuated error-function connection of Eq. (43). As expected from a comparison of the plots in Fig. 1, the AC curves based on $w_{\lambda}^{\mathrm{g}}$ in panes (g)-(i) are more sharply peaked and more highly localized than those based on $w_{\lambda}^{\mathrm{e}}$ in panes (d)-(f). Gaussian attenuation yields AC curves that vanish in both limits. Otherwise, the behavior is similar to that of the error-function curves-as $Z$ increases, the peak in the connection rises and moves to the right. Although, we note the presence of two peaks in the correlation curves.

\section{The hydrogen molecule}

Having explored dynamic correlation in the high-density limit for two-electron systems in Sec. III C, we now explore the transition from dynamic correlation to static correlation by stretching the $\mathrm{H}_{2}$ molecule. The $\mathrm{H}_{2}$ molecule is a prototypical system that can be considered as representative for the dissociation of electron-pair bonds in general. The fully interacting wave function $\Psi_{1}$ is a singlet at all geometries, ${ }^{84}$ consistent with $\rho_{\alpha}(\mathbf{r})=\rho_{\beta}(\mathbf{r})=\rho(\mathbf{r}) / 2$, as is imposed in spinrestricted Kohn-Sham theory. In the present work, all calculations use the restricted formalism. For a more detailed discussion, see Ref. 22. We now examine the AC curves for the bond lengths $R=0.7,1.4,3.0,5.0,7.0$, and 10.0 (in units of $a_{0}$ ) for the different choices of two-electron interaction in Eqs. (41)-(43). In all calculations, the aug-cc-pVQZ basis set $^{79-81}$ is used.

The $\mathrm{H}_{2}$ energy and its components are listed in Table III, in the same way as for the helium series in Table II. At equilibrium, $H_{\mathrm{s}}[v]$ is $2 \%$ lower than $T_{\mathrm{s}}[\rho]+(v \mid \rho), H_{\mathrm{c}}[v]$ is three times larger than $T_{\mathrm{c}}[\rho]$, and $\int \mathcal{W}_{\lambda}[v] \mathrm{d} \lambda$ is $9 \%$ larger than $\int \mathcal{W}_{\lambda}[\rho] \mathrm{d} \lambda$. These results are similar to that of the he- lium atom $(Z=2)$ in Table II. However, as $R$ increases, the differences between the two connections become smaller until they vanish in the dissociation limit. Thus, at $R=10 a_{0}$, $T_{\mathrm{c}}[\rho]$ and $H_{\mathrm{c}}[v]$ are both smaller than $0.001 E_{\mathrm{h}}$; in the dissociation limit, these two correlation corrections vanish and $T_{\mathrm{s}}[\rho]+(v \mid \rho)=H_{\mathrm{s}}[v]$. Concerning the two-electron interactions, we note that $\int \mathcal{W}_{\lambda}[\rho] \mathrm{d} \lambda \approx \int \mathcal{W}_{\lambda}[v] \mathrm{d} \lambda \approx 1 / R$ at large $R$; at $R=10 a_{0}$, this relation holds to within $0.001 E_{\mathrm{h}}$. At this separation, $\int \mathcal{W}_{\lambda}[\rho] \mathrm{d} \lambda$ consists of a dominant positive Coulomb interaction $J[\rho]=0.725 E_{\mathrm{h}}$ of which $0.625 E_{\mathrm{h}}$ is selfinteraction, which is subsequently cancelled by exchange $E_{\mathrm{x}}[\rho]=-J[\rho] / 2=-0.362 \quad E_{\mathrm{h}}$ and static correlation $E_{\mathrm{c}}[\rho]$ $=-0.262 E_{\mathrm{h}}$.

In Fig. 5, we have plotted $\mathcal{W}_{\lambda}^{\mathrm{s}}[v]$ and $\mathcal{W}_{\lambda}^{\mathrm{s}}[\rho]$ in pane (a), $\mathcal{W}_{\lambda}^{\mathrm{e}}[v]$ and $\mathcal{W}_{\lambda}^{\mathrm{e}}[\rho]$ in pane (b), and $\mathcal{W}_{\lambda}^{\mathrm{g}}[v]$ and $\mathcal{W}_{\lambda}^{\mathrm{g}}[\rho]$ in pane (c). For the short bond distances $R=0.7 a_{0}$ and $1.4 a_{0}$, the shape of the AC curves $\mathcal{W}_{\lambda}^{\mathrm{s}}[\rho]$ and $\mathcal{W}_{\lambda}^{\mathrm{s}}[v]$ in pane (a) are similar to those for helium in pane (a) of Fig. 3 and are indicative of the quadratic dependence of dynamical correlation energy on $\lambda$. However, as the bond is stretched, the curves in pane (a) bend more sharply, the changes as a function of $\lambda$ becoming localized to the low- $\lambda$ end of the curves. At $R=10 a_{0}$, the density- and potential-fixed AC curves coincide (following the above discussion). Except for very small coupling strengths, the two curves are horizontal at $0.100 E_{\mathrm{h}}$, reflecting the complete absence of dynamical correlation in this system.

Turning our attention to the range-separated AC curves in panes (b) and (c) of Fig. 5, we note how long-range interactions become more dominant with increasing separation $R$. This behavior is particularly pronounced for the Gaussianattenuated error-function curves, which develop a semidiscontinuity at $\lambda \approx 0.43$ for $R=10.0 a_{0}$. For $\lambda>0.43$, the $w_{\lambda}^{g}$ operator only samples interactions between electrons located less than about $8 a_{0}$ apart. Since there are few such interactions in a system consisting of two hydrogen atoms $10 a_{0}$ apart, the $\mathcal{W}_{\lambda}[\rho]$ curve drops to zero around $\lambda=0.43$. Comparing the density- and potential-fixed curves in panes (b) and (c), we note that peaks in the potential-fixed curves appear at slightly larger $\lambda$ values, reflecting the more compact electron density in the potential-fixed case for $\lambda<1$. Finally, we note that the end points in the range-separated curves are the same as for the helium series in Fig. 3, being dictated by the number of electrons.

We now turn our attention to Fig. 6, where we have plotted the total density-fixed AC curves and their exchangecorrelation and correlation contributions for $\mathrm{H}_{2}$, in the same manner as for the helium series in Fig. 4. We consider first 


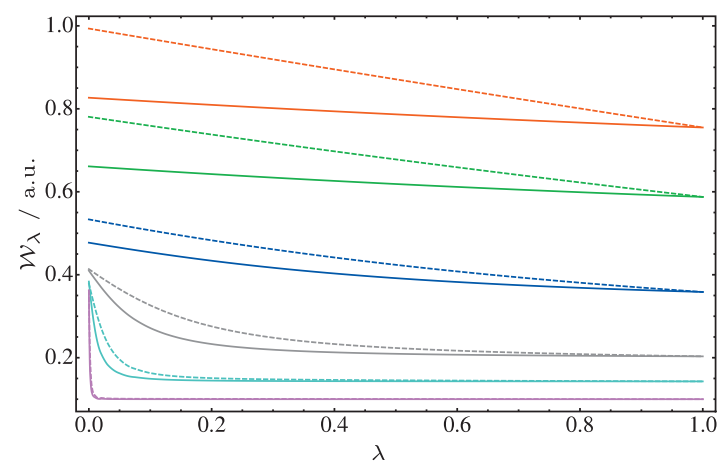

(a)

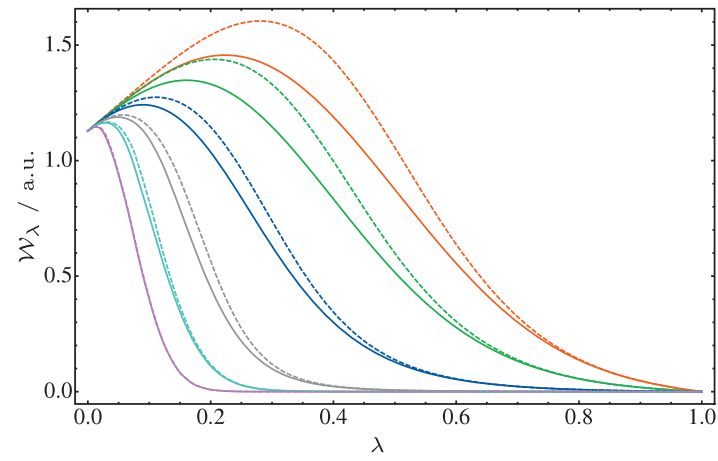

(b)

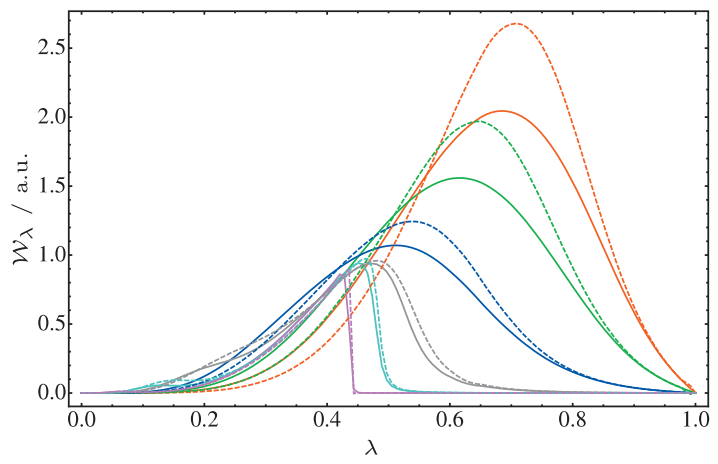

(c)

FIG. 5. AC curves (atomic units) $\mathcal{W}_{\lambda}[\rho]$ (full lines) and $\mathcal{W}_{\lambda}[v]$ (dashed lines) for $\mathrm{H}_{2}$ with $R=0.7 a_{0}, 1.4 a_{0}, 3.0 a_{0}, 5.0 a_{0}, 7.0 a_{0}$, and $10.0 a_{0}$ for $w_{\lambda}^{\mathrm{s}}$ in pane (a), for $w_{\lambda}^{\mathrm{e}}$ in pane (b), and for $w_{\lambda}^{\mathrm{g}}$ in pane (c). In all panes, the curves fall with increasing $R$ at $\lambda=0.8$.

the range-independent curves in the first row. In pane (a), $\mathcal{W}_{\lambda}^{\mathrm{s}}[\rho]$ has been plotted in the same manner as in Fig. 4; here, the AC curves fall with increasing $R$. In pane (b), we have removed the Coulomb contribution and plotted $\mathcal{W}_{\mathrm{xc}, \lambda}^{\mathrm{s}}[\rho]$. Since the Coulomb and exchange contributions decrease in magnitude with increasing $R$, while the correlation contribution increases, the curves now cross but may be distinguished by noting that $\mathcal{W}_{\mathrm{xc}, 0}^{\mathrm{s}}[\rho]$ increases with increasing $R$. Finally, in pane (c), the exchange contribution has also been removed, illustrating how the correlation contribution $\mathcal{W}_{\mathrm{c}, \lambda}^{\mathrm{s}}[\rho]$ increases in magnitude as the bond is stretched. At dissociation, correlation is entirely static and $T_{\mathrm{c}, 1}[\rho]=0$, noting that the area between a horizontal line at the $\mathcal{W}_{\mathrm{c}, 1}^{\mathrm{s}}[\rho]$ value and the curve represents $T_{\mathrm{c}, 1}[\rho]$. At dissociation, the curve would fall immediately to the exchange energy of a hydrogen atom and then become horizontal, as is required to cancel the spurious self-interaction energy in a restricted formalism.

The second row of Fig. 6 shows the range-separated AC curves corresponding to the error-function attenuated operator in Eq. (42) for the various geometries of $\mathrm{H}_{2}$. It is noteworthy that for the exchange-correlation curve $\mathcal{W}_{\mathrm{xc}, \lambda}^{\mathrm{e}}[\rho]$ in pane (e), oscillations begin to appear in the low $\lambda$ regime for the two longest bond lengths. As for the helium series in Fig. 4, we have transformed the AC into a form with known end points but a more complicated path. Subtraction of the exchange contribution yields the correlation curves $\mathcal{W}_{\mathrm{c}, \lambda}^{\mathrm{e}}[\rho]$ in pane (f). For $R=0.7$ and $1.4 a_{0}$, the curves are similar to the helium curves in Fig. 4. As we stretch the bond the density becomes more diffuse, the peak becomes more negative, and moves to the left, analogous to the behavior for descending nuclear charge in the helium isoelectronic series. The interpretation of this behavior was given in Sec. III C and similar arguments apply here. As static correlation becomes dominant in pane (f) of Fig. 6, the area under the curve becomes larger and spreads over more of the $\lambda$ range with large contributions below $\lambda=0.3$, which were essentially negligible at short bond lengths, where dynamic correlation is dominant.

In the last row of Fig. 6, the curves corresponding to the Gaussian-attenuated error-function connection in Eq. (43) are presented. For short bond distances, the AC curves are simple in structure; as the bond stretches, the curves become more complicated as semidiscontinuities and oscillations develop. At $R=10 a_{0}$, the total AC curve $\mathcal{W}_{\lambda}^{\mathrm{g}}[\rho]$ vanishes for $\lambda \geq 0.43$, reflecting the physical separation of the electrons in two atoms. By contrast, the $\mathcal{W}_{\mathrm{xc}, \lambda}^{\mathrm{g}}[\rho]$ and $\mathcal{W}_{\mathrm{c}, \lambda}^{\mathrm{g}}[\rho]$ curves are more complicated, illustrating how the total AC curve $\mathcal{W}_{\lambda}^{\mathrm{g}}[\rho]$ vanishes for large $\lambda$ by an intricate cancellation of classical Coulomb with non-classical Coulomb contributions that arise from exchange and static correlation.

\section{E. The relevance of range-dependent ACs to range-separated approaches}

The density-fixed ACs presented here correspond to the link from the noninteracting Kohn-Sham system to the physical interacting system. In the range-separated approaches proposed by Savin, ${ }^{28}$ one instead begins from a partially interacting system by decomposing the universal density functional as

$$
F_{\lambda}^{\kappa}[\rho]=F_{\kappa}[\rho]+\int_{\kappa}^{\lambda} \mathcal{W}_{\nu}[\rho] \mathrm{d} \nu,
$$

where $F_{\kappa}[\rho]$ with $0 \leq \kappa \leq \lambda$ is defined as before in Eq. (5), whereas $\mathcal{W}_{\nu}[\rho]=F_{\nu}^{\prime}[\rho]$ is given in Eq. (18). We may further decompose the last term in Eq. (49) in the usual manner, into Coulomb and exchange-correlation contributions

$$
\int_{\kappa}^{\lambda} \mathcal{W}_{\nu}[\rho] \mathrm{d} \nu=J_{\lambda}^{\kappa}[\rho]+E_{\mathrm{xc}, \lambda}^{\kappa}[\rho]
$$

where by analogy with Eqs. (24) and (30) we have defined

$$
J_{\lambda}^{\kappa}[\rho]=\frac{1}{2} \iint\left[w_{\lambda}\left(r_{12}\right)-w_{\kappa}\left(r_{12}\right)\right] \rho\left(\mathbf{r}_{1}\right) \rho\left(\mathbf{r}_{2}\right) \mathrm{d} \mathbf{r}_{1} \mathrm{~d} \mathbf{r}_{2},
$$




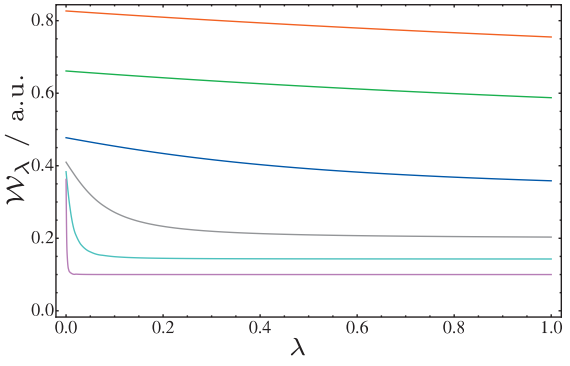

(a)

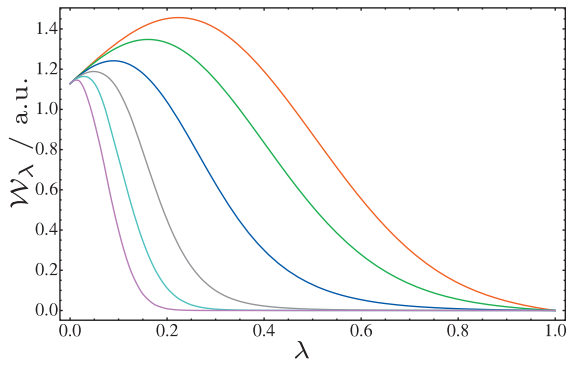

(d)

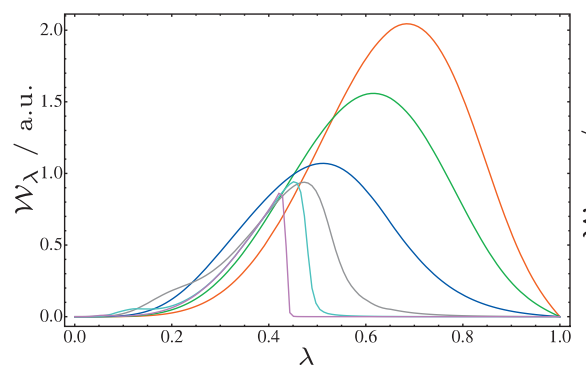

(g)

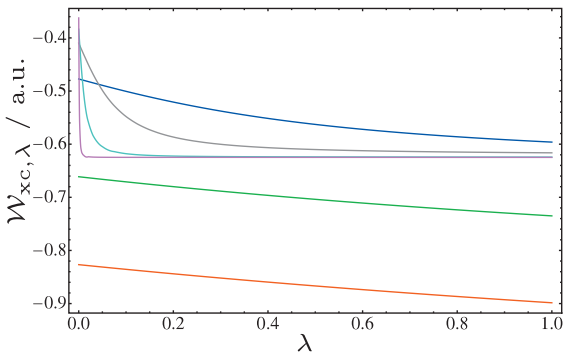

(b)

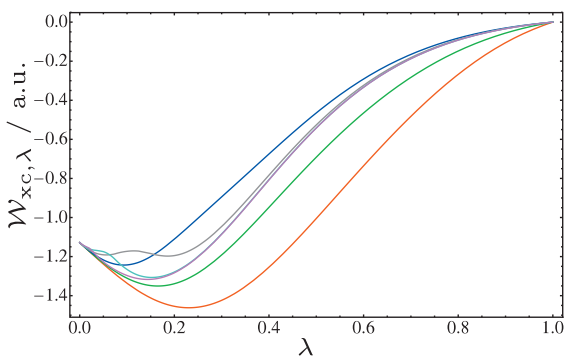

(e)

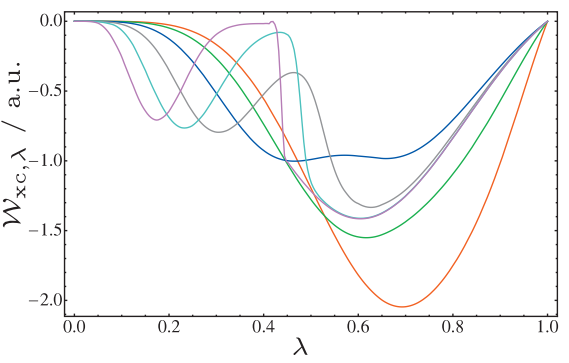

(h)

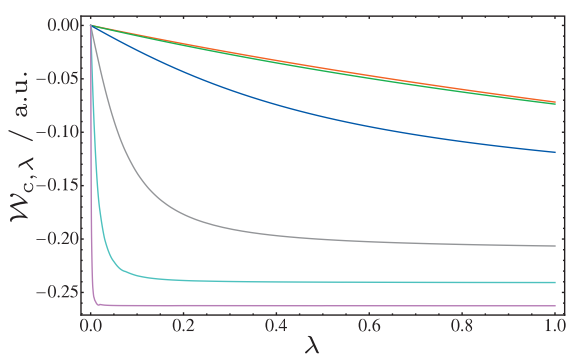

(c)

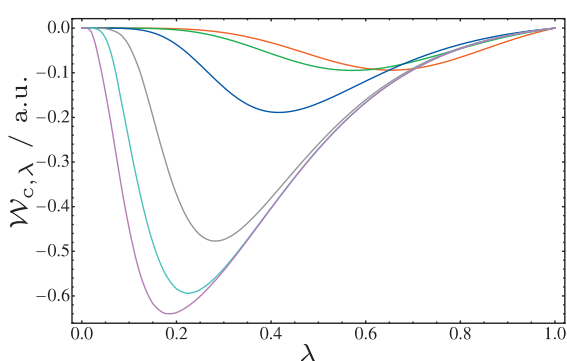

(f)

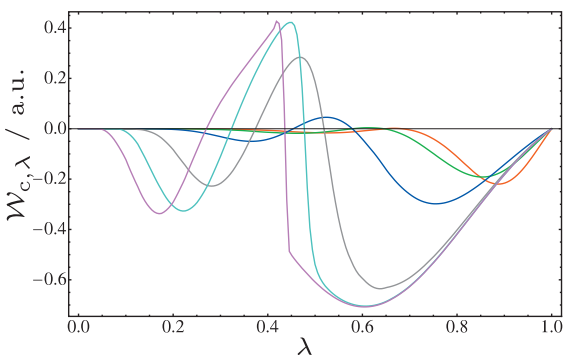

(i)

FIG. 6. AC curves (atomic units) for $\mathrm{H}_{2}$ at nuclear separations $R=0.7 a_{0}, 1.4 a_{0}, 3.0 a_{0}, 5.0 a_{0}, 7.0 a_{0}$, and $10.0 a_{0}$ for $w_{\lambda}^{\mathrm{s}}$ in panes (a)-(c), for $w_{\lambda}^{\mathrm{e}}$ in panes (d)-(f), and for $w_{\lambda}^{\mathrm{g}}$ in panes $(\mathrm{g})-(\mathrm{i})$. For each $\mathrm{AC}$, we have plotted the total curve $\mathcal{W}_{\lambda}[\rho]$ to the left, the exchange-correlation curve $\mathcal{W}_{\mathrm{xc}, \lambda}[\rho]$ in the middle, and the correlation curve $\mathcal{W}_{\mathrm{c}, \lambda}[\rho]$ to the right. In panes $(\mathrm{a}),(\mathrm{d})$, and $(\mathrm{g})$, the curves fall with increasing $R$ at $\lambda=0.8$; in the other panes, the curves may be distinguished by noting that the same color scheme is used in all panes.

$$
E_{\mathrm{xc}, \lambda}^{\kappa}[\rho]=\int_{\kappa}^{\lambda} \mathcal{W}_{\mathrm{xc}, \nu[}[\rho] \mathrm{d} \nu
$$

When $w_{\kappa}\left(r_{12}\right)$ represents the long-range Coulomb operator, then $J_{\lambda}^{\kappa}[\rho]$ and $E_{\mathrm{xc}, \lambda}^{\kappa}[\rho]$ are the short-range Coulomb and exchange-correlation contributions, respectively, calculated from the short-range $w_{\lambda}\left(r_{12}\right)-w_{\kappa}\left(r_{12}\right)$ operator.

The short-range contributions of interest in rangeseparated approaches are therefore obtained simply by choosing a value of $\lambda$ [or equivalently $\mu$; see the discussion below Eq. (46)] greater than zero to define a partially interacting system with a long-range component of the twoelectron interaction. Integration over the remainder of the $\mathcal{W}_{\lambda}[\rho]$ AC from this value to $\lambda>\kappa$ gives the short-range complement to the Coulomb and exchange-correlation energies. Subtraction of the short-range Coulomb and/or exchange contributions gives the corresponding exchangecorrelation or correlation-only ACs. The ACs of the present work are therefore of direct relevance to range-separated methodologies, the AC path being defined by the choice of long-range interaction, typically chosen to be the erf or erf- gau attenuated operators as have been examined here. Many other choices of long-range interaction are of course possible-see, for example, the work of Baer and Neuhauser ${ }^{85}$ who recently used the generalized AC with an alternative form for the interaction to establish a class of density functionals with correct long-range asymptotic behavior. A similar division can, of course, also be made for the standard linear AC, although the resulting hybrid theories would not emphasize the range of the interaction, only the strength of the interactions due to the uniform manner in which the interactions are introduced with $\lambda$, see Fig. 1. Analogous hybrid theories can also be constructed for the potential-fixed case for each choice of the two-electron interaction.

The behavior of the generalized ACs in Fig. 6 suggests that static and dynamic correlation are not easily divided by range-separated operators, as attempted in hybrid short-range DFT and long-range wave function approaches. Whereas dynamic correlation in $\mathrm{H}_{2}$ is predominantly a short-range effect, static correlation arises from a complicated interplay of short- and long-range interactions, as illustrated in panes (f) 
and (i) in Fig. 6. This observation rationalizes to some extent the success of range-separated approaches in treating dispersion (long-range dynamic correlation is missing in local and semilocal DFT) and their failure in describing bond dissociation (where static correlation dominates).

We note that in pane (f) of Fig. 6 the error-function AC for the correlation energy becomes small for the values of $\lambda$ in the range $0.25<\lambda<0.3$ for the two shortest bond lengths. This corresponds to $\mu=\lambda /(1-\lambda)$ in the range $0.33<\mu$ $<0.42$, consistent with the optimal values delivered by the prescription of Fromager et al., ${ }^{37,38}$ which are typically in the range $0.3<\mu<0.4$, depending on the system considered. Furthermore, these values are consistent with those used in the LC functionals of Hirao et al. ${ }^{40}$ and in the CAM-B3LYP functional, ${ }^{44,45}$ in which only the exchange contribution to the functional is range separated, the correlation being treated in a standard manner. Since the correlation parts of these functionals cannot describe long-range dynamic correlation or static correlation but do account for short-range dynamic correlation, it is perhaps unsurprising that the optimization of $\mu$ for molecules close to their equilibrium geometries also results in a value in this range. Such values of $\mu$ improve the description of the long-range exchange contributions while larger values would compromise the description of short-range dynamic correlation which is dominant for these systems. From the figures presented in the present work, we expect the optimal $\mu$ value to depend strongly on the compactness or diffuseness of the electronic density and as such this value should not be a fixed parameter, an idea which was recently explored by Krukau et al. ${ }^{86}$ and Henderson et al. ${ }^{87}$ However, the implementation of such functionals is much more involved.

\section{CONCLUSIONS}

We have examined the AC for generalized, rangedependent two-electron interactions, in potential- and density-fixed formulations. The helium isoelectronic series and the stretching of the hydrogen molecule were investigated. These prototypical systems exhibit a range of densities (diffuse and compact) and types of correlation (dynamic and static). In each case, the total energy was decomposed into its individual components, comparing quantities relevant in PFT and DFT. Standard, range-independent AC curves were compared with range-dependent curves obtained by attenuating the two-electron interaction with the error function and with a Gaussian-attenuated error function. For the helium isoelectronic series, the range-dependent ACs displayed a peak that moved to large values of the interaction strength $\lambda$ as the density became more compact; these peaks being most compact for the Gaussian-attenuated error function. For $\mathrm{H}_{2}$, this peak moved to smaller $\lambda$ values as the bond was stretched and static correlation began to dominate; the striking feature of the Gaussian-attenuated curves being the development of a semidiscontinuity for large bond distances $R$.

The density-fixed ACs were then further decomposed into their exchange-correlation and correlation-only contributions. For the helium series, the exchange-correlation ACs were similar to the negative of the full ACs (owing to the fact that the exchange interaction is dominant in these systems and equal to minus one-half of the Coulomb contribution). For $\mathrm{H}_{2}$, a similar situation was observed for small internuclear distances. However, as the bond was stretched, the ACs exhibited a more complicated structure due to the increasing role of static correlation.

Perhaps the most instructive ACs in the present work are those corresponding to the correlation energy. For the standard AC of the helium isoelectronic series, the shape of these curves was understood by noting that as $Z$ increased, the correlation energy could be described more and more accurately by the second-order Görling-Levy perturbation theory. For the range-dependent ACs, the curves revealed that correlation interactions in these systems are shorter ranged than the Coulomb and exchange interactions. In addition, as $Z$ increased, the correlation contributions became localized to the high- $\lambda$ end of the connection, consistent with the shortranged interactions in the increasingly compact densities.

For small internuclear separations, the $\mathrm{H}_{2}$ correlationonly AC curves were similar to those of the helium atom. With increasing $R$ and increasing static correlation, the standard connection bent more sharply until, at dissociation, it would drop immediately from zero to the exchange energy of a hydrogen atom and remain at this value for all $\lambda>0$. With increasing $R$ and increasing importance of longer-range interactions, the corresponding error-function curve displayed a broad peak that moved to smaller $\lambda$ values but without localizing at this end of the connection. For the Gaussianattenuated error-function connection, the correlation AC displayed a complicated structure, evolving a semidiscontinuity as $R$ increased. Both observations are consistent with the fact that the role of static correlation is to account for deficiencies arising from the use of a single determinant to describe the system and as such cannot be localized to a specific range. For $\mathrm{H}_{2}$ in a restricted formalism, this deficiency is manifested by the spurious self-interaction error. Implications for the description of static correlation by range-separated approaches were discussed in Sec. III E.

In the present work, we have utilized the error-functionbased operators to modify the electronic interaction, rather than to attempt its separation. The extent to which this approach is helpful for the development of exchangecorrelation functionals for practical use by modeling these connections in a similar manner to the approach in Ref. 88 is being investigated. However, the ability to relate the features of these integrands to features of the electronic densities, in addition to their end points corresponding to known constants, would seem to offer some promise. Of the two alternatives considered in the present work, the error-functionbased connection appears to be the better candidate for future development of functionals based on modeling the AC integrand owing to the relatively smooth behavior of its correlation integrand.

\section{ACKNOWLEDGMENTS}

The authors thank Wim Klopper, A. Savin, and P. GoriGiorgi for useful discussions. This work has been supported by the CoE Centre for Theoretical and Computational Chem- 
istry through Grant No. 179568/V30. A.M.T. gratefully acknowledges support from the Norwegian Research Council through Grant Nos. 171185/V30 and 197446/V30 and the hospitality of the Università degli Studi di Trieste during a visit for part of this work.

${ }^{1}$ J. Harris and R. O. Jones, J. Phys. F: Met. Phys. 4, 1170 (1974).

${ }^{2}$ D. C. Langreth and J. P. Perdew, Solid State Commun. 17, 1425 (1975).

${ }^{3}$ O. Gunnarsson and B. I. Lundqvist, Phys. Rev. B 13, 4274 (1976).

${ }^{4}$ O. Gunnarsson and B. I. Lundqvist, Phys. Rev. B 15, 6006 (1977).

${ }^{5}$ D. C. Langreth and J. P. Perdew, Phys. Rev. B 15, 2884 (1977).

${ }^{6}$ A. D. Becke, J. Chem. Phys. 98, 1372 (1993).

${ }^{7}$ J. P. Perdew, M. Ernzerhof, and K. Burke, J. Chem. Phys. 105, 9982 (1996).

${ }^{8}$ M. Ernzerhof, Chem. Phys. Lett. 263, 499 (1996).

${ }^{9}$ M. Ernzerhof, J. P. Perdew, and K. Burke, Int. J. Quantum Chem. 64, 285 (1997).

${ }^{10}$ K. Burke, M. Ernzerhof, and J. P. Perdew, Chem. Phys. Lett. 265, 115 (1997).

${ }^{11}$ M. Seidl, J. P. Perdew, and S. Kurth, Phys. Rev. Lett. 84, 5070 (2000).

${ }^{12}$ M. Seidl, Int. J. Quantum Chem. 91, 145 (2003).

${ }^{13}$ M. Fuchs, Y. M. Niquet, X. Gonze, and K. Burke, J. Chem. Phys. 122 094116 (2005).

${ }^{14}$ P. Mori-Sánchez, A. J. Cohen, and W. Yang, J. Chem. Phys. 124, 091102 (2006).

${ }^{15}$ A. J. Cohen, P. Mori-Sánchez, and W. Yang, J. Chem. Phys. 127, 034101 (2007).

${ }^{16}$ A. J. Cohen, P. Mori-Sánchez, and W. Yang, J. Chem. Phys. 126, 191109 (2007).

${ }^{17}$ D. P. Joubert and G. P. Srivastava, J. Chem. Phys. 109, 5212 (1998).

${ }^{18}$ F. Colonna and A. Savin, J. Chem. Phys. 110, 2828 (1999).

${ }^{19}$ D. Frydel, W. M. Terilla, and K. Burke, J. Chem. Phys. 112, 5292 (2000).

${ }^{20}$ A. Savin, F. Colonna, and M. Allavena, J. Chem. Phys. 115, 6827 (2001)

${ }^{21}$ R. Pollet, F. Colonna, T. Leininger, H. Stoll, H.-J. Werner, and A. Savin, Int. J. Quantum Chem. 91, 84 (2003).

${ }^{22}$ A. M. Teale, S. Coriani, and T. Helgaker, J. Chem. Phys. 130, 104111 (2009)

${ }^{23}$ Q. Wu and W. Yang, J. Chem. Phys. 118, 2498 (2003).

${ }^{24}$ E. H. Lieb, Int. J. Quantum Chem. 24, 243 (1983).

${ }^{25}$ W. Yang, P. W. Ayers, and Q. Wu, Phys. Rev. Lett. 92, 146404 (2004).

${ }^{26}$ E. K. U. Gross and C. R. Proetto, J. Chem. Theory Comput. 5, 844 (2009).

${ }^{27}$ W. Yang, J. Chem. Phys. 109, 10107 (1998).

${ }^{28}$ A. Savin, in Recent Developments and Applications of Modern Density Functional Theory, edited by J. M. Seminario (Elsevier, Amsterdam, 1996), p. 327.

${ }^{29}$ J. Toulouse, A. Savin, and H.-J. Flad, Int. J. Quantum Chem. 100, 1047 (2004).

${ }^{30}$ J. Toulouse, F. Colonna, and A. Savin, J. Chem. Phys. 122, 014110 (2005).

${ }^{31}$ E. Goll, H.-J. Werner, and H. Stoll, Phys. Chem. Chem. Phys. 7, 3917 (2005).

${ }^{32}$ E. Goll, H. J. Werner, H. Stoll, T. Leininger, P. Gori-Giorgi, and A. Savin, Chem. Phys. 329, 276 (2006).

${ }^{33}$ E. Goll, M. Ernst, F. Moegle-Hofacker, and H. Stoll, J. Chem. Phys. 130, 234112 (2009)

${ }^{34}$ J. G. Ángyán, I. C. Gerber, A. Savin, and J. Toulouse, Phys. Rev. A 72, 012510 (2005)

${ }^{35}$ T. Leininger, H. Stoll, H. J. Werner, and A. Savin, Chem. Phys. Lett. 275, 151 (1997).

${ }^{36}$ R. Pollet, A. Savin, T. Leininger, and H. Stoll, J. Chem. Phys. 116, 1250 (2002).

${ }^{37}$ E. Fromager, J. Toulouse, and H. J. Aa. Jensen, J. Chem. Phys. 126, 074111 (2007)

${ }^{38}$ E. Fromager, F. Réal, P. Wåhlin, U. Wahlgren, and H. J. Aa. Jensen, J. Chem. Phys. 131, 054107 (2009).

${ }^{39}$ E. Fromager, R. Cimiraglia, and H. J. Aa. Jensen, Phys. Rev. A 81, 024502 (2010).

${ }^{40}$ H. Iikura, T. Tsuneda, T. Yanai, and K. Hirao, J. Chem. Phys. 115, 3540 (2001).

${ }^{41}$ J.-W. Song, S. Tokura, T. Sato, M. A. Watson, and K. Hirao, J. Chem. Phys. 127, 154109 (2007).
${ }^{42}$ J. Heyd, G. E. Scuseria, and M. Ernzerhof, J. Chem. Phys. 118, 8207 (2003).

${ }^{43}$ J. Heyd and G. E. Scuseria, J. Chem. Phys. 120, 7274 (2004).

${ }^{44}$ T. Yanai, D. P. Tew, and N. C. Handy, Chem. Phys. Lett. 393, 51 (2004).

${ }^{45}$ M. J. G. Peach, T. Helgaker, P. Sałek, T. W. Keal, O. B. Lutnaes, D. J. Tozer, and N. C. Handy, Phys. Chem. Chem. Phys. 8, 558 (2006).

${ }^{46}$ T. M. Henderson, A. F. Izmaylov, G. E. Scuseria, and A. Savin, J. Chem. Phys. 127, 221103 (2007).

${ }^{47}$ R. D. Adamson, J. P. Dombroski, and P. M. W. Gill, Chem. Phys. Lett. 254, 329 (1996).

${ }^{48}$ P. M. W. Gill and R. D. Adamson, Chem. Phys. Lett. 261, 105 (1996).

${ }^{49}$ P. M. W. Gill, R. D. Adamson, and J. A. Pople, Mol. Phys. 88, 1005 (1996).

${ }^{50}$ J. Toulouse, F. Colonna, and A. Savin, Mol. Phys. 103, 2725 (2005).

${ }^{51}$ M. Levy, Proc. Natl. Acad. Sci. U.S.A. 76, 6062 (1979).

${ }^{52}$ M. Levy, Phys. Rev. A 26, 1200 (1982).

${ }^{53} \mathrm{H}$. Eschrig, The Fundamentals of Density Functional Theory, 2nd ed. (Eagle, Leipzig, 2003).

${ }^{54}$ O. Sinanoğlu, Phys. Rev. 122, 493 (1961).

${ }^{55}$ A. Görling and M. Levy, Phys. Rev. B 47, 13105 (1993)

${ }^{56}$ A. Görling and M. Levy, Phys. Rev. A 50, 196 (1994).

${ }^{57}$ J. Toulouse, F. Colonna, and A. Savin, Phys. Rev. A 70, 062505 (2004).

${ }^{58}$ C. C. M. Samson, W. Klopper, and T. Helgaker, Comput. Phys. Commun. 149, 1 (2002).

${ }^{59}$ See: http://www.kjemi.uio.no/software/dalton/dalton.html for DALTON, a molecular electronic structure program, release 2.0, 2005.

${ }^{60}$ S. Hirata, S. Ivanov, I. Grabowski, R. J. Bartlett, K. Burke, and J. D. Talman, J. Chem. Phys. 115, 1635 (2001).

${ }^{61}$ R. J. Bartlett, I. Grabowski, S. Hirata, and S. Ivanov, J. Chem. Phys. 122, 034104 (2005).

${ }^{62}$ V. N. Staroverov, G. E. Scuseria, and E. R. Davidson, J. Chem. Phys. 125, 081104 (2006).

${ }^{63}$ V. N. Staroverov, G. E. Scuseria, and E. R. Davidson, J. Chem. Phys. 124, 141103 (2006).

${ }^{64}$ D. Rohr, O. V. Gritsenko, and E. J. Baerends, J. Mol. Struct.: THEOCHEM 762, 193 (2006).

${ }^{65}$ A. Heßelman, A. W. Götz, F. Della Sala, and A. Görling, J. Chem. Phys. 127, 054102 (2007).

${ }^{66}$ C. Kollmar and M. Filatov, J. Chem. Phys. 127, 114104 (2007).

${ }^{67}$ D. P. Joubert, J. Chem. Phys. 127, 244104 (2007).

${ }^{68}$ C. Kollmar and M. Filatov, J. Chem. Phys. 128, 064101 (2008).

${ }^{69}$ T. Heaton-Burgess, F. A. Bulat, and W. Yang, Phys. Rev. Lett. 98, 256401 (2007).

${ }^{70}$ T. Heaton-Burgess and W. Yang, J. Chem. Phys. 129, 194102 (2008).

${ }^{71}$ M. J. G. Peach, J. A. Kattirtzi, A. M. Teale, and D. J. Tozer, J. Phys. Chem. A 114, 7179 (2010).

${ }^{72}$ F. A. Bulat, T. Heaton-Burgess, A. J. Cohen, and W. Yang, J. Chem. Phys. 127, 174101 (2007).

${ }^{73}$ A. Halkier, T. Helgaker, P. Jørgensen, W. Klopper, H. Koch, J. Olsen, and A. K. Wilson, Chem. Phys. Lett. 286, 243 (1998)

${ }^{74}$ C. J. Umrigar and X. Gonze, Phys. Rev. A 50, 3827 (1994).

${ }^{75}$ A. A. Jarzęcki and E. R. Davidson, Phys. Rev. A 58, 1902 (1998).

${ }^{76}$ P. M. W. Gill, D. L. Crittenden, D. P. O'Neill, and N. A. Besley, Phys. Chem. Chem. Phys. 8, 15 (2006).

${ }^{77}$ J. Katriel, S. Roy, and M. Springborg, J. Chem. Phys. 123, 104104 (2005).

${ }^{78}$ J. P. Perdew, E. R. Mc Mullen, and A. Zunger, Phys. Rev. A 23, 2785 (1981).

${ }^{79}$ T. H. Dunning, Jr., J. Chem. Phys. 90, 1007 (1989).

${ }^{80}$ R. A. Kendall, T. H. Dunning, Jr., and R. J. Harrison, J. Chem. Phys. 96, 6796 (1992).

${ }^{81}$ D. E. Woon and T. H. Dunning, Jr., J. Chem. Phys. 100, 2975 (1994).

${ }^{82}$ D. E. Woon and T. H. Dunning, Jr., J. Chem. Phys. 103, 4572 (1995).

${ }^{83}$ M. Levy and J. P. Perdew, Phys. Rev. A 32, 2010 (1985).

${ }^{84}$ W. Kolos and C. C. J. Roothaan, Rev. Mod. Phys. 32, 219 (1960).

${ }^{85}$ R. Baer and D. Neuhauser, Phys. Rev. Lett. 94, 043002 (2005).

${ }^{86}$ A. V. Krukau, G. E. Scuseria, J. P. Perdew, and A. Savin, J. Chem. Phys. 129, 124103 (2008).

${ }^{87}$ T. M. Henderson, B. G. Janesko, G. E. Scuseria, and A. Savin, Int. J. Quantum Chem. 109, 2023 (2009).

${ }^{88}$ A. M. Teale, S. Coriani, and T. Helgaker, J. Chem. Phys. 132, 164115 (2010). 